\title{
Preclinical efficacy, safety, and immunogenicity of PHH-1V, a second- generation COVID-19 vaccine candidate based on a novel recombinant RBD fusion heterodimer of SARS-CoV-2.
}

Antonio Barreiro*1, Antoni Prenafeta*1, Gregori Bech-Sabat ${ }^{1}$, Mercè Roca ${ }^{1}$, Eva Perozo ${ }^{1}$, Ricard March $^{1}$, Luis González ${ }^{1}$, Laia Madrenas ${ }^{1}$, Júlia Corominas ${ }^{1}$, Àlex Fernández ${ }^{1}$, Mercè Molas ${ }^{1}$, Thais Pentinat ${ }^{1}$, Clara Panosa ${ }^{1}$, Alberto Moreno ${ }^{1}$, Ester Puigvert ${ }^{1}$, Eva Pol ${ }^{1}$, Jordi Palmada ${ }^{1}$, Carme Garriga ${ }^{1}$, Teresa Prat ${ }^{1}$, Júlia Vergara-Alert ${ }^{2}$, Cristina Lorca-Orón ${ }^{2}$, Núria Roca ${ }^{2}$, Leira FernándezBastit $^{2}$, Jordi Rodon ${ }^{2}$, Mònica Pérez ${ }^{2}$, Joaquim Segalés ${ }^{3,4}$, Edwards Pradenas ${ }^{5}$, Silvia Marfil ${ }^{5}$, Benjamin Trinité ${ }^{5}$, Raquel Ortiz ${ }^{5}$, Bonaventura Clotet ${ }^{5,6}$, Julià Blanco ${ }^{5,6}$, Jorge Díaz Pedroza ${ }^{7}$, Rosa Ampudia Carrasco ${ }^{7}$, Yaiza Rosales Salgado 7 , Jordina Loubat-Casanovas ${ }^{7}$, Sara Capdevila Larripa ${ }^{7}$, Julia Garcia Prado 7,5 , Jordi Barretina ${ }^{7}$, Marta Sisteré-Oró ${ }^{8}$, Paula Cebollada Rica ${ }^{8}$, Andreas Meyerhans ${ }^{8,9}$, Laura Ferrer ${ }^{1}$

\footnotetext{
*: A. Barreiro and A. Prenafeta contributed equally to this work.

${ }^{1}$ HIPRA. Avda. La Selva, 135. 17170 Amer (Girona), Spain.
}

${ }^{2}$ Institut de Recerca i Tecnologia Agraroalimentàries (IRTA), Centre de Recerca en Sanitat Animal (CReSA, IRTA-UAB), Campus de la UAB, 08193 Cerdanyola del Vallès, Spain.

3Universitat Autònoma de Barcelona, CReSA (IRTA-UAB), Campus de la UAB, 08193 Cerdanyola del Vallès, Spain.

${ }^{4}$ Departament de Sanitat i Anatomia Animals, Facultat de Veterinària, UAB, 08193 Cerdanyola del Vallès, Spain.

5IrsiCaixa. AIDS Research Institute, Germans Trias i Pujol Research Institute (IGTP), Can Ruti Campus, UAB, 08916 Badalona, Spain.

6University of Vic-Central University of Catalonia (UVic-UCC), 08500, Vic, Catalonia, Spain.

${ }^{7} \mathrm{CMCiB}-\mathrm{IGTP}$. Comparative Medicine and Bioimage Centre of Catalonia, Germans Trias i Pujol Research Institute. 08916 Badalona, Spain.

8Infection Biology Laboratory, Department of Experimental and Health Sciences, Universitat Pompeu Fabra (UPF), 08003 Barcelona, Spain.

9ICREA, Catalan Institution for Research and Advanced Studies Pg. Lluís Companys 23, 08010 Barcelona, Spain. 


\begin{abstract}
Since the genetic sequence of SARS-CoV-2 became available in January 2020, new vaccines have been developed at an unprecedented speed. The current vaccines have been directly associated with a decline in new infection rates, prevention of severe disease and an outstanding decrease in mortality rates. However, the pandemic is still far from being over. New Variants of Concern (VoCs) are continuously evolving. Thus, it is essential to develop accessible second-generation COVID-19 vaccines against known and future VoCs to mitigate the current pandemic. Here, we provide preclinical data showing the immunogenicity, efficacy, and safety results in mice of a receptor-binding domain (RBD)-based recombinant protein vaccine candidate (PHH-1V) which consists of a novel RBD fusion heterodimer containing the B.1.1.7 (alpha) and B.1.351 (beta) variants of SARS-CoV-2, formulated with an oil-based adjuvant equivalent to MF59C.1. BALB/C and K18-hACE2 mice were immunized with different doses of recombinant RBD fusion heterodimer, following a two-dose prime-and-boost schedule. Upon 20 $\mu \mathrm{g}$ RBD fusion heterodimer/dose immunization, BALB/c mice produced RBD-binding antibodies with neutralising activity against the alpha, beta, gamma, and delta variants. Furthermore, vaccination elicited robust activation of $\mathrm{CD}^{+}$and $\mathrm{CD} 8^{+} \mathrm{T}$ cells with early expression of Th1 cytokines upon in vitro restimulation, along with a good tolerability profile. Importantly, vaccination with $10 \mu \mathrm{g}$ or $20 \mu \mathrm{g}$ RBD fusion heterodimer/dose conferred $100 \%$ efficacy preventing mortality and bodyweight loss upon SARS-CoV-2 challenge in K18-hACE2 mice. These findings demonstrate the feasibility of this novel recombinant vaccine strategy, allowing the inclusion of up to 2 different RBD proteins in the same vaccine. Most importantly, this new platform is easy to adapt to future VoCs and has a good stability profile, thus ensuring its global distribution.
\end{abstract}

Keywords: SARS-CoV-2, COVID-19, second-generation vaccines, immunology, recombinant RBD fusion heterodimer, B.1.1.7 (alfa), B.1.351 (beta), VoCs, preclinical, mice. 
bioRxiv preprint doi: https://doi.org/10.1101/2021.11.22.469117; this version posted December 4, 2021. The copyright holder for this

\section{Introduction}

In December 2019, the Severe Acute Respiratory Syndrome Coronavirus-2 (SARS-CoV-2) was identified as the etiological agent of the novel Coronavirus disease 2019 (COVID-19). Soon after that, the scientific community and the pharmaceutical industry began focusing on the development of effective COVID-19 vaccines to mitigate the health emergency. Thanks to these efforts, several vaccines are currently available, and more than 7 billion doses have been administered worldwide (November 2021) (WHO, 2021). The decline in new infection rates in many countries coincides with the introduction of vaccines. However, COVID-19 cases continue to emerge, probably due to the appearance and evolution of novel SARS-CoV-2 variants, the decline of immunological protection provided by the current vaccines, and, especially, the lack of homogenous distribution of COVID-19 vaccines, with only $4.4 \%$ of people in low-income countries having received at least one dose (November 2021) (Hannah Ritchie et al., 2020; Mathieu et al., 2021). As the global outbreak continues, the pandemic is far from being over, and it is not clear if the available vaccines will be sufficient to revert the situation. Thus, it is still critical to develop second-generation vaccines using different platforms that are effective against novel variants and that could be further used as a booster, particularly to maintain or even enhance immunity against SARS-CoV-2 (Günl et al., 2021; Moyo-Gwete et al., 2021). Moreover, it is of relevance that these novel vaccines can be stored in refrigerated conditions, making them easier to distribute, avoiding lower and less available extremely low storage temperature conditions to ensure their global supply. Currently, authorised vaccines, either approved under emergency use or with full license, and vaccine candidates are based on viral vectored approaches, inactivated viruses, nucleic acid-based vaccines, and subunit vaccines.

The SARS-CoV-2 is a novel betacoronavirus, which belongs to the subfamily Coronovirinae within the family Coronaviridae and the order Nidovirales. The SARS-CoV-2 genome is a single-stranded positive-sense RNA (+ssRNA) molecule. The genome size ranges between 27 and $32 \mathrm{kbp}$, one of the largest known RNA viruses. The genomic structure of SARS-CoV-2 contains at least six Open Reading Frames (ORFs), encoding for at least four structural proteins, namely: envelope or spike (S) glycoprotein S; membrane (M) proteins, responsible for the shaping of the virions; envelope (E) proteins, responsible for the virions assembly and release; and nucleocapsid (N) proteins, involved in the RNA genome packaging (Alanagreh, Alzoughool and Atoum, 2020). The trimeric $S$ glycoprotein of SARS-CoV-2 is the primary target of viral neutralising antibodies and has been the main protein candidate for vaccine development (Vogel et al., 2020). Consistent with SARS$\mathrm{CoV}$, angiotensin-converting enzyme 2 (ACE2) binding of the $\mathrm{S}$ protein allows cellular entry of SARS-CoV-2 viral particles (Zhou et al., 2020). This protein consists of 2 domains, S1 and S2, allowing the binding of the viral particles and the cellular entry by fusing with the host cell membrane (Huang et al., 2020). The receptor binding domain (RBD) (Thr333-Gly526) is found in the $\mathrm{S} 1$ domain, and it contains a highly immunogenic receptor binding motif (RBM) that directly interacts with ACE2 and neutralising antibodies (Yi et al., 2020). Therefore, the majority of key mutations are found in the RBM, allowing the virus adapting to the previous developed immunity. To date, several SARS-CoV-2 VoCs with key mutations in the $S$ protein have emerged: alpha (B.1.1.7), beta (B.1.351), gamma (P.1) and delta (B.1.617.2) (Plante et al., 2021).

The $S$ protein is the primary target in vaccine development against betacoronaviruses due to its accessibility for immune recognition (Dai and Gao, 2021). It has been reported that two proline substitutions in the original $S$ protein sequence (S-2P) of MERS-CoV, SARS-CoV and HKU1 coronavirus maintain the antigenic conformation (Pallesen et al., 2017). Thus, learning from these previous results, this $\mathrm{S}-2 \mathrm{P}$ design is used in the authorised mRNA-based vaccines Comirnaty ${ }^{\circledR}$ (Pfizer-BioNTech) (Comirnaty SmPC, 2021) and Spikevax ${ }^{\circledR}$ (Moderna) (Spikevax SmPC, 2021), which substitute the residues $\mathrm{K} 986$ and V987 for prolines from the original $\mathrm{S}$ 
bioRxiv preprint doi: https://doi.org/10.1101/2021.11.22.469117; this version posted December 4, 2021. The copyright holder for this preprint (which was not certified by peer review) is the author/funder. All rights reserved. No reuse allowed without permission.

protein variant. Likewise, the adenoviral vector-based vaccine Janssen (Johnson \& Johnson) also contains DNA-encoding for the S-2P protein of the SARS-CoV-2 (COVID-19 Vaccine Janssen, 2021).

Adjuvanted protein-based subunit vaccines represent an important type of vaccines, yet their development has lagged compared to other platforms due to the need to optimise the manufacturing process for each protein antigen. The most advanced subunit vaccine programme against COVID-19 is the Novavax vaccine candidate, (NVX-CoV-2373), which is produced in insect cells in combination with a saponin-based adjuvant (Matrix-M) (Keech et al., 2020). This vaccine candidate consists of the stable full-length S protein in the antigenically optimal prefusion conformation. In addition, the Sanofi-GSK vaccine candidate consists of soluble prefusion-stabilized S trimers from SARS-CoV-2 produced in insect cells with the ASO3 adjuvant (Francica et al., 2021). Both vaccine candidates are currently being tested in human clinical trials (Keech et al., 2020b; Goepfert et al., 2021). Notably, recombinant proteins are competitive vaccine candidates with an adequate safety profile, no risk of genome integration, no live components, and suitable for people with compromised immune systems (Kyriakidis et al., 2021), showing high productivity yields and good stability profiles (Gavi Alliance- Covax collaboration platform, 2021; Kleanthous et al., 2021; Kyriakidis et al., 2021).

The majority of cloned neutralising antibodies target the RBD in the S1 domain (Piccoli et al., 2020), although there are additional immunogenic epitopes outside this domain (Wang et al, 2020). Considering that the RBD domain of the S protein directly interacts with the ACE2 receptor, and therefore, RBD-targeting antibodies are not expected to cause AntibodyDependent Enhancement (ADE), unlike non-neutralising or sub-neutralising antibodies (Lee et al., 2020), these data highlight the importance of RBD in the immune response against SARSCoV-2.

Given the inherent particularities of the S protein, and especially the RBD domain, our team developed a vaccine-candidate platform based on this immunogen. Among the tested preliminary vaccine-candidates, combined with one or several adjuvants, we finally proceeded with a protein-based subunit vaccine candidate, namely $\mathrm{PHH}-1 \mathrm{~V}$, consisting of a recombinant RBD fusion heterodimer of the B.1.1.7 and B.1.351 variants of SARS-CoV-2 produced in Chinese Hamster Ovary (CHO) cells with an oil-based adjuvant equivalent to MF59C.1. Specifically, MF59C. 1 is an oil-in-water emulsion adjuvant that stimulates the immune response, the safety of which is well-known in humans since approximately 100 million doses have been already distributed to populations including pregnant women and children (Ko and Kang, 2018). Thus, the main aims of this study were to assess the safety and efficacy of the PHH-1V vaccine in BALB/c and K18-hACE2-transgenic mice models, and to characterise the RBD fusion heterodimer antigen and its immunogenicity.

\section{Results}

\subsection{Recombinant RBD fusion heterodimer expression and characterisation}

The antigen of the PHH-1V vaccine candidate is the SARS-CoV-2 virus recombinant S protein RBD fusion heterodimer consisting of the B.1.351 and B.1.1.7 variants. This recombinant subunit antigen is a RBD fusion heterodimer antigen formed by two monomers (Figure $1(\mathbf{A})$ ). The $\mathrm{N}$ terminal monomer contains the amino acid sequence of the SARS-CoV-2 RBD protein from the B.1.351 (beta) variant, whereas the C-terminal monomer contains the homologous sequence from the B.1.1.7 (alpha) variant. According to the structural modelling, the critical mutations involved in the higher affinity towards the human ACE2- receptor and the potential immune escape of both variants are clearly exposed on the protein surface. The heterodimer is expressed in mammalian $\mathrm{CHO}$ cells and is used with an oil-in-water adjuvant equivalent to MF59C.1. After expressing the antigen in a bioreactor fed-batch cultivation, it is purified by a complex 
downstream process consisting of sequential stages, including depth and tangential filtration, chromatography steps, and sterile filtrations. The final product is a highly purified antigen, as determined by SDS-PAGE and SEC-HPLC (Figure 1 (B, C), suitable for vaccine formulation. Surface plasmon resonance (SPR) analysis showed an affinity constant for hACE2 of $0.099 \mathrm{nM}$ (Figure 1 (D)).

\subsection{Recombinant RBD fusion heterodimer antigen immunogenicity}

\subsubsection{RBD-specific binding antibody titres}

The mouse strain B6.Cg-Tg(K18-ACE2)2PrImn/J (K18-hACE2) (Jackson Laboratories, ME, USA) and BALB/C (Envigo, IN, USA) were immunized with different doses of the recombinant RBD fusion heterodimer antigen. The prime immunization with the $\mathrm{PHH}-1 \mathrm{~V}$ candidate induced higher titres of RBD binding antibodies in the groups $C$ to $F$ compared to the control (group $A$ ) on day 21 post-first immunization (D21) $(p<0.05)$ (Figure 2(A)).

After the prime-boost immunization, all vaccinated groups ( $B$ to $F$ ) reached higher IgG titres than the control group on D35/D37 (14/16 after the boost; $p<0.01)$. At D35/D37, specific SARS-CoV2 RBD-binding antibodies were detected in groups $B$ to $D$ in a dose-dependent manner, with significant differences among these groups $(p<0.01)$. However, no significant differences were observed among the groups immunized with more than $1 \mu \mathrm{g}$ of recombinant RBD fusion heterodimer antigen (groups D to F). Thus, the IgG response was saturated from $1 \mu \mathrm{g}$ immunization. Likewise, the RBD-specific binding antibody titres were also increased in SARSCoV-2 infected K18-hACE2 mice upon vaccination (group $C$ and D) compared to infected and non-infected PBS-vaccinated mice (group A and B) (Figure S1). Both vaccinated groups had similar RBD-specific antibody titres and were vaccinated with 10 or $20 \mu \mathrm{g}$ of recombinant protein RBD fusion heterodimer, respectively.

To estimate the type of cellular immune response elicited by the vaccine, the $\lg \mathrm{g} 2 \mathrm{a} / \operatorname{lgG} 1$ ratios were calculated as a surrogate of the Th1/Th2 cellular immune response. The IgG2a/IgG1 ratio of groups $E$ and $F$ was 0.74 and 0.75 , respectively, which suggests a balanced Th1/Th2 immunogenic response upon $\mathrm{PHH}-1 \mathrm{~V}$ vaccination in mice (Figure 2(B)).

\subsubsection{SARS-CoV-2 neutralising antibody titres}

SARS-CoV-2 neutralising antibodies titres in sera from BALB/c mice were determined by Pseudovirus-based neutralisation assay (PBNA) against the $S$ protein of different variants on D35/D37 (14/16 days after the boost). Prime-boost immunization of groups C to $F$ induced higher neutralising antibody titres against the $S$ protein of the alpha variant compared to the control group $A(p<0.01)$ (Figure $3(A))$. No neutralising antibody response was observed in group $B$, although IgG binding antibodies were detected on D35/D37. The mean neutralising antibody titres observed in groups $C$ to $E$ remained the same since no statistically significant differences were observed. However, vaccination with $20 \mu \mathrm{g}$ of RBD fusion heterodimer antigen (group F) induced higher neutralising titres than groups $C$ and $D$. In fact, high neutralising titres against all the tested variants (alpha, beta, gamma, and delta) were detected in sera from group $\mathrm{F}$ (Figure 3(B)), especially for the alpha and beta variants. On the other hand, the SARS-CoV-2 neutralising antibodies against the original variant (Wuhan) were increased in SARS-CoV-2 infected humanized K18-hACE2 mice upon vaccination (groups $C$ and D) compared to infected and noninfected PBS-vaccinated mice (groups A and B) (Figure S2). Notably, the levels of neutralising antibodies were similar between both vaccinated groups.

\subsubsection{Cellular immune response upon $P H H-1 V$ vaccination of $B A L B / c$ mice}

The characterization of the antigen-specific response of splenic T cells 14-16 days after the boost immunization was performed by intracellular cytokine staining (ICS) and enzyme-linked 
bioRxiv preprint doi: https://doi.org/10.1101/2021.11.22.469117; this version posted December 4, 2021. The copyright holder for this

immunospot (ELISpot) assays in female BALB/c mice from groups $A$ (control), E and $F$ (vaccinated with $5 \mu \mathrm{g}$ or $20 \mu \mathrm{g}$ of recombinant protein RBD fusion heterodimer, respectively). The ICS data indicate that upon the stimulation with an RBD peptide pool, splenocytes from group F displayed significant activation of $C D 4^{+} T$ cells expressing IFN- $-(p<0.01)$ and IL-2 $(p<0.05)$ compared to the control group (Figure 4 (A)). No significant antigen-specific response of $C D 4^{+} T$ cells expressing TNF- $\alpha$ or IL-4 was observed in group F when compared to the control group. Notably, immunization of mice with a lower RBD dose (group E) did not induce IFN- $\gamma$, TNF- $\alpha$, IL-2 or IL-4 producers' memory $\mathrm{CD} 4^{+} \mathrm{T}$ cells after the in vitro restimulation.

Furthermore, splenocytes from group $\mathrm{F}$ showed significant activation of $\mathrm{CD} 8^{+} \mathrm{T}$ cells expressing IFN- $\gamma(p<0.05)$ and IL-2 $(p<0.01)$ after the antigen-specific restimulation ( $>0.1 \%$ activation) compared to the control group (Figure $4(B)$ ). No $\mathrm{CD}^{+} \mathrm{T}$ cell response was observed in splenocytes from group $E$ compared to the control group. Hence, considering the $T$ cells expressing any of the analysed Th1 cytokines (IFN- - , TNF- $\alpha$ and IL-2), group F showed a higher $\mathrm{CD} 4^{+} \mathrm{T}$ cell response than the control group $(p<0.05)$, and a higher percentage of animals showed activated $\mathrm{CD} 8^{+} \mathrm{T}$ cells $(p<0.05)(>0.25 \%$ activation) (Figure $\mathbf{S 3}$ ).

The IFN- $\gamma$ and IL-4 ELISpot assays showed no significant differences between the two doses of recombinant protein RBD fusion heterodimer (group E vs group F) (Figure 5). However, both groups showed a higher percentage of IFN $-\gamma^{+}$and IL- $4^{+}$spots compared to the control group $(p<0.01)$. Importantly, the percentage of IFN- $\gamma^{+}$and $\mathrm{IL}-4^{+}$in group $\mathrm{F}$ was similar, denoting a balanced Th1/Th2 response, while the percentage of $\mathrm{IL}-4^{+}$spots was significantly higher than IFN- $\gamma^{+}$spots in group $\mathrm{E}(p<0.01)$, suggesting a Th2-biased response in mice immunised with $5 \mu \mathrm{g}$ of recombinant protein RBD fusion heterodimer.

The extracellular cytokine levels were measured by Luminex Multiplex in supernatants from splenocytes stimulated with a pool of peptides from SARS-CoV-2 RBD. The levels of IL-2 $(p<0.05)$, IL-5 $(p<0.01)$ and TNF- $\alpha(p<0.05)$ were higher in the supernatants from group E splenocytes compared to the control group (Figure 6). Similarly, the levels of IL-5 $(p<0.01)$ and TNF- $\alpha(p<0.05)$ were statistically higher in group $\mathrm{F}$ compared to group $\mathrm{A}$. A tendency towards an increase in the levels of IL-2 ( $p=0.062)$ was also observed in group F compared to group A.

\subsection{Recombinant RBD fusion heterodimer antigen efficacy in K18-hACE2 mice}

To analyse the protective efficacy of the vaccine candidate PHH-1V against COVID-19 and the pathogenic outcomes derived from the SARS-CoV-2 infection, K18-hACE2 mice were used as a challenge model. All groups were vaccinated intramuscularly following the two-dose prime-andboost schedule: $1^{\text {st }}$ dose (prime) at D0 and $2^{\text {nd }}$ dose (boost) at D21. The SARS-CoV-2 challenge was performed on a subset of animals at D35 through intranasal infection.

\subsubsection{Clinical signs and weights post-challenge}

The primary endpoint reporting the protective capacity of the vaccine candidates was weight loss and/or mortality post-challenge. Survival curves are presented in Figure 7 (A). Clinical signs of the SARS-CoV-2 infection were observed only in the non-vaccinated and infected group (B) on days 5 ( 3 animals) and 6 ( 3 animals) post-challenge. In all cases, clinical signs led to end-point criteria and the animals were euthanised. Thus, survival curves were significantly different among the different groups $(p<0.001)$. The weekly individual body weights of each group during the vaccination period and post-challenge are shown in Figures S4 (B) and $\mathbf{7}(\mathbf{B})$, respectively. Animals of group B experienced a remarkable weight loss from D3 post-challenge onwards, as expected due to the COVID-19 infection, showing a significantly lower weight compared with vaccinated animals from group D on D5 post-challenge $(p<0.05)$ and from groups C and D on D6 post-challenge $(p<0.01)$. 


\subsubsection{Viral load by genomic $R T-q P C R$}

Total viral RNA was determined in oropharyngeal samples (Figure 8 (A)), lungs, nasal turbinate, brain (Figure $\mathbf{8}(\mathbf{B})$ ), trachea, pharynx, spleen and heart (Figure $\mathbf{8}(\mathbf{C})$ ). Viral RNA was determined by quantitative real-time quantitative polymerase chain reaction (RT-qPCR) at D37 (2 days postinfection), D39 (4 days post-infection), D42 (in males, 7 days post-infection) and D43 (in females, 8 days post-infection), or at the time of euthanasia in animals reaching end-point criteria before the scheduled euthanasia day. As it can be seen in Figure 8 (A, B, C), immunization with both doses of PHH-1V (10 $\mu \mathrm{g}$ or $20 \mu \mathrm{g}$ of recombinant protein RBD fusion heterodimer), reduced the viral load measured by PCR in oropharyngeal swabs, lungs, nasal turbinate, brain, trachea, pharynx, spleen and heart after the experimental infection compared to the infected control (group B).

\subsubsection{Virus titration from tissue samples determined in Vero E6 cells}

Virus titres were determined using a standard $50 \%$ tissue culture infectious dose $\left(\mathrm{TCID}_{50}\right)$ assay on positive samples of qPCR. No infectious virus was detected in oropharyngeal swabs, pharynx, spleen and trachea. Regarding heart samples, only one out of the three positive samples by RTqPCR resulted positive in viral isolation in cell culture (group B at D4). For the other organs, the results are shown in Figure 9. For the analysed tissues, the samples from group $D$ had a significant lower infective viral load at D2 post-challenge and a tendency towards a decrease compared to group B at D7. Likewise, the group $C$ showed a tendency towards a decrease in the infective viral load compared to group B at D2 post-challenge.

\subsubsection{Histopathology}

Histopathological analyses were determined in the nasal turbinate, lungs, and brain for all animals. In other non-main target tissues of the virus (spleen, trachea, or heart), 2 animals per group were analysed. No lesions were found in the spleen, trachea, and heart of any of the studied animals. As it can be seen in Figure 10, considering data from all days, the infected nonvaccinated control (group B) had a higher histopathological score in the lungs compared to group $C(p<0.05)$ and in the brain compared to group $C(p<0.005)$ and $D(p<0.005)$. No significant differences between groups were observed in the histopathological score of the nasal turbinates.

\subsection{Safety of the recombinant RBD fusion heterodimer antigen after vaccination}

The preclinical safety of the PHH-1V candidate vaccine was evaluated on BALB/c mice immunised with different doses of the RBD fusion heterodimer, by measuring the bodyweight of each animal once a week until D35/D37. For additional safety information, clinical signs and local reactions were monitored. No differences in the bodyweight were observed between the different dose groups (Figure S4 (A)), and no clinical signs or local reactions were detected after the vaccinations in $B A L B / C$ mice.

Additionally, the PHH-1V safety was evaluated in humanised K18-hACE2 mice and bodyweight and clinical signs were also monitored during the vaccination period. As observed with the $\mathrm{BALB} / \mathrm{C}$ mice, no significant changes in the bodyweight were observed between the different groups (Figure S4 (B)) and vaccinated animals did not show clinical signs or local reactions. The histological evaluation in the injection site revealed a mild lesion, with multifocal mononuclear inflammatory infiltrates within and around muscular fibres, in one of the hind limbs of 1 animal vaccinated with $10 \mu \mathrm{g}$ RBD fusion heterodimer/dose at D2 and 2 animals vaccinates with $20 \mu \mathrm{g}$ RBD fusion heterodimer/dose at D4 post-challenge (Figure 10). 
bioRxiv preprint doi: https://doi.org/10.1101/2021.11.22.469117; this version posted December 4, 2021. The copyright holder for this

\section{Discussion}

In this study, the effect of the recombinant protein RBD fusion heterodimer dose on the immunogenicity and safety of the PHH-1V vaccine was tested in BALB/c and K18-hACE2 mice. Furthermore, the preclinical efficacy of the vaccine candidate was also assessed in SARS-CoV-2infected and non-infected K18-hACE2 mice. We show that the active substance of the PHH-1V vaccine candidate, the RBD fusion heterodimer, is stable and has an affinity constant of 0.099 $\mathrm{nM}$ against the human ACE2 receptor, which indicates an outstanding binding affinity with its natural ligand. The whole sequence of the antigen originates from the SARS-CoV-2 RBD domains of the B.1.1.7 (alpha) and B.1.351 (beta) variants, which have been shown by Ramanathan et al. (2021) to bind ACE2 with increased affinity. We were able to obtain the antigen at high purity, which is consistent with its use as an active drug substance in a vaccine. $\mathrm{CHO}$, the expression system selected to produce this antigen, has been a workhorse for decades for the production of monoclonal antibodies and other protein-based therapeutic entities (Sharker and Rahman, 2021). It has been clearly accepted by regulatory agencies worldwide for this purpose.

The $\mathrm{PHH}-1 \mathrm{~V}$ vaccine candidate was shown to be safe in mice since the tested doses did not cause clinical signs (general and local) nor bodyweight loss in either immunised BALB/c or K18-hACE2 mice. Although the histological evaluation of the injection sites revealed mild lesions with cellular infiltrates in few vaccinated animals, these were attributable to the local innate immune response induced upon injection with adjuvant-containing vaccines (Singh M, O'Hagan, 1999) We consistently observed an adequate safety profile in other animal species in which the $\mathrm{PHH}$ $1 \mathrm{~V}$ vaccine candidate has been tested, such as rats, rabbits, cynomolgus monkeys and pigs (unpublished data). The oil-based adjuvant equivalent to MF59C.1 used in this vaccine might be related to the good tolerability shown in animal models, as it is based on an adjuvant that is widely regarded as safe in humans (O'Hagan et al., 2013).

Regarding the RBD-binding antibodies humoral response, a dose-response was observed at D35/D37 upon vaccination with RBD heterodimer doses of $0.04,0.2$ and $1 \mu \mathrm{g} / \mathrm{dose}$, however, this response saturates with higher immunization doses. Significant total IgG titres were observed even after one dose for doses of $0.2 \mu \mathrm{g}$ and above. These results suggest a good potency profile for antigen included in the $\mathrm{PHH}-1 \mathrm{~V}$ vaccine candidate, with similar or superior performance to other previously reported immunogens based on similar platforms (Figure 2 (A)) (Kuo et al., 2020; Tian et al., 2021). Moreover, potent pseudovirus-neutralising activity against the alpha variant was elicited by $0.2 \mu \mathrm{g}$ RBD fusion heterodimer/dose immunization, reaching the highest titres with the $20 \mu \mathrm{g}$ RBD fusion heterodimer/dose immunization. Furthermore, a robust pseudovirus-neutralising activity of sera from mice immunised with $20 \mu \mathrm{g}$ of RBD fusion heterodimer/dose was confirmed against the beta, gamma, and delta variants. This crossreactivity was previously confirmed in earlier exploratory trials, where no significant differences were observed in the pseudovirus-neutralising titres against the alpha, beta, and gamma variants in mice upon vaccination with $20 \mu \mathrm{g}$ RBD fusion heterodimer/dose (data not shown). Notably, the pseudovirus neutralization assays from this work were performed in the same laboratory and under the exact same conditions as those that were previously reported to have a good correlation with live virus neutralization assays (Trinité et al., 2021), which highlights the biological relevance of the obtained neutralising antibody titres that were obtained. Previous results show that upon vaccination with mRNA-based vaccines (Spikevax ${ }^{\circledR}$ and Comirnaty ${ }^{\circledR}$ ), the SARS-CoV-2 neutralization capacity is significantly decreased against the gamma and beta variants, even in fully vaccinated individuals. This reduction in neutralization capacity is similar to the delta variant (García-Beltran et al., 2021; Planas et al., 2021; Wall et al., 2021). Thus, even though the recombinant protein RBD fusion heterodimer was designed to elicit a response against the SARS-CoV-2 alpha and beta variants, our data also demonstrate a further neutralising activity against the novel delta variant, which is currently dominant around the world to date (Hadfield et al., 2018; Genomic epidemiology of novel coronavirus - Global subsampling., 2021). 
bioRxiv preprint doi: https://doi.org/10.1101/2021.11.22.469117; this version posted December 4, 2021. The copyright holder for this preprint (which was not certified by peer review) is the author/funder. All rights reserved. No reuse allowed without permission.

This is critical to mitigating the current pandemic situation, although further studies will have to confirm our findings in human subjects. Moreover, our antigen contains several mutations that are of high concern, mutations which are present in currently designated VoCs and which could potentially arise in future variants. That includes the E484K substitution present in beta and the more recently emerged B.1.621 (mu) variant. E484K is related to immune escape and reduced antibody neutralization, compromising the efficacy of currently approved vaccines (Jangra et al., 2021).

In regard to the cellular response upon vaccination, the ICS data indicate that immunization with the highest dose of $20 \mu \mathrm{g}$ RBD fusion heterodimer/dose induced a robust Th1-dominant response with activation of $\mathrm{CD}^{+}$and $\mathrm{CD}^{+} \mathrm{T}$ cells expressing IFN- $\gamma$ and IL-2. Notably, no significant IL-4 expression was detected by ICS in the splenocytes from immunised animals. However, the expression of this Th2 cytokine was detected by IL-4 ELISpot assays in splenocytes from both immunised groups. Specifically, according to the ELISpot results, the immunization with $5 \mu \mathrm{g}$ RBD fusion heterodimer/dose elicited a Th2-biased response, while the immunization with $20 \mu \mathrm{g}$ RBD fusion heterodimer promoted a balanced Th1/Th2 response. These differences in the cytokine expression between both assays might be explained by the differences in the incubation time of the splenocytes after the RBD peptide pool stimulation, which was $48 \mathrm{~h}$ for the ELIspot compared to $5 \mathrm{~h}$ for the ICS. Furthermore, the experimental conditions (number of splenocytes and incubation time) assayed to detect IFN- $\gamma$ and IL-4 via ELISpot were different; hence, these data have to be interpreted carefully. It is known that, for most infections, Th1 immunity is protective since it promotes humoral immunity, and phagocytic and cytotoxic $T$ cell activity, whereas the Th2 response assists with the resolution of inflammation (Spellberg and Edwards, 2001). Based on the ICS data, the immunization with $20 \mu \mathrm{g}$ RBD fusion heterodimer/dose seems to induce a polarised Th1 immune response.

Extracellular cytokine production was also measured by Luminex Multiplex in supernatants from splenocytes after $48 \mathrm{~h}$ of stimulation, where a balanced production of Th1 (TNF- $\alpha$, IL-2) and Th2 (IL-5 but no IL-4 nor IL-6) cytokines was found in vaccinated mice. Notably, IFN- $y$ was not detected by Luminex, probably due to the early expression of this factor and its rapid degradation. Importantly, IL-10 was not detected in the supernatants, which indicates that the immunization with $\mathrm{PHH}-1 \mathrm{~V}$ did not elicit an anti-inflammatory response after the re-stimulation of splenocytes with RBD peptide pools.

The IgG2a/IgG1 ratio was measured for the assessment of the Th1/Th2 polarization after the prime-boost immunization. IgG1 is produced during any type of immune response, while IgG2a is mainly produced during a Th1-polarised immune response (Snapper and Paul, 1987). Mice immunised with either $5 \mu \mathrm{g}$ or $20 \mu \mathrm{g}$ RBD fusion heterodimer/dose induced RBD-binding antibodies of both IgG2a and IgG1 subclasses, with an IgG2a/IgG1 ratio near 0.8 , indicating a balanced Th1-Th2 response upon PHH-1V vaccination.

Thus, all the data suggest that PHH-1V immunization with $20 \mu \mathrm{g} \mathrm{RBD}$ fusion heterodimer/dose elicits a robust $\mathrm{CD} 4^{+}$and $\mathrm{CD} 8^{+} T$ cell response with an early expression of Th1 cytokines upon restimulation in vitro, and balanced Th1-Th2 cytokine production after $48 \mathrm{~h}$ post-stimulation.

Regarding the preclinical efficacy of the PHH-1V vaccine candidate, it was tested at 2 different doses, $10 \mu \mathrm{g}$ and $20 \mu \mathrm{g}$ RBD fusion heterodimer/doses in K18-hACE2 mice. Upon the SARS-CoV2 challenge, vaccinated animals were able to overcome the infection since neither clinical signs nor bodyweight loss was detected. Contrarily, all non-vaccinated and infected animals reached the end-point criteria at D5 or D6 post-challenge and had to be euthanised. Furthermore, this group of animals experienced a remarkable weight loss from D3 post-challenge onwards due to the SARS-CoV-2 infection. Therefore, our data show $100 \%$ efficacy in preventing mortality and body weight loss in infected K18-hACE2 mice upon PHH-1V vaccination. 
bioRxiv preprint doi: https://doi.org/10.1101/2021.11.22.469117; this version posted December 4, 2021. The copyright holder for this

In addition, immunization with either the $10 \mu \mathrm{g}$ or the $20 \mu \mathrm{g}$ RBD fusion heterodimer/dose of $\mathrm{PHH}-1 \mathrm{~V}$ reduced the viral load measured via qPCR in the lungs, nasal turbinate and brain in K18hACE2 mice. The viral load excretion measured in oropharyngeal swabs was also reduced upon vaccination. Moreover, differences in the viral load after the SARS-CoV-2 challenge between vaccinated animals and infected non-vaccinated control animals were also found in other respiratory (trachea and pharynx) and systemic (spleen and heart) organs. Notably, when RTqPCR positive samples were titrated to determine the infective viral load, most of the samples of vaccinated animals showed negative results, whereas, most of the samples of the infected control group resulted in significantly higher viral loads. Taken together, these results suggest less viral replication in vaccinated mice, which discards antibody-dependent enhancement (ADE) of the infection upon vaccination. Indeed, it is known that RBD poses a low potential for risk of ADE because antibodies against this domain block receptor binding (An et al., 2021). Likewise, the histopathological evaluation of tissues from vaccinated mice showed no lesions in the brain and mild lesions in the lungs and nasal turbinate upon SARS-CoV- 2 infection. Contrarily, infected control mice displayed moderate lesions in the lungs and brain, which is consistent with the high viral loads detected in this group.

Overall, in this study, the PHH-1V vaccine has been shown to be safe and immunogenic in mice, inducing RBD-binding and neutralising antibodies. Mice immunised with $20 \mu \mathrm{g}$ of recombinant protein RBD fusion heterodimer/dose showed neutralising activity against the alpha, beta, gamma and delta variants. Likewise, immunization with $20 \mu \mathrm{g}$ of RBD fusion heterodimer/dose elicited robust activation of $\mathrm{CD} 4^{+}$and $\mathrm{CD} 8^{+} \mathrm{T}$ cells, producing an early Th1 response upon in vitro re-stimulation. Importantly, vaccination with either $10 \mu \mathrm{g}$ or $20 \mu \mathrm{g}$ RBD fusion heterodimer/dose prevented weight loss and clinical signs (including mortality) upon SARS-CoV-2 challenge in mice. Both tested doses reduced viral loads in several organs and prevented the infective viral load in the lungs and brain upon the experimental infection. In addition, immunization with $20 \mu \mathrm{g}$ of RBD recombinant protein fusion dimer reduced the infective viral load in the upper respiratory tract (nasal turbinate). Most importantly, besides the efficacy and safety features of PHH-1V, this second-generation COVID-19 vaccine is easy to adapt to potential emergent SARS-CoV-2 variants, allowing for the inclusion of up to 2 different RBDs to generate cross-immunity against novel emergent variants. The $\mathrm{PHH}-1 \mathrm{~V}$ vaccine candidate showed promising preclinical data and is currently being evaluated in a Phase I/Ila clinical trial (NCT05007509) (U.S. National Library of Medicine, 2021).

\section{Methods}

\subsection{Recombinant RBD fusion heterodimer expression and characterisation}

The antigen was produced in a bioreactor based on a selected stable $\mathrm{CHO}-c l o n e . ~ A$ fed-batch strategy was used for high cell density cultivation and expression of the RBD fusion heterodimer. Upon harvest, the cell broth was clarified by depth filtration. The clarified supernatant was further purified via sequential chromatography. The purified antigen was then buffer exchanged by tangential flow filtration and filter sterilised. Purity and integrity were evaluated by SDS-PAGE with Bolt ${ }^{\mathrm{TM}} 4$ to $12 \%$, Bis-Tris gels (ThermoFisher, ref. NW04120BOX), stained with one-step blue protein gel stain (Biotium, ref. 21003), and by SEC-HPLC with an xbridge protein BEH SEC (Waters, ref. 186009160) connected to a HP1100 system (Agilent Technologies).

The affinity test of the RBD dimer with human ACE2 by surface plasmon resonance (SPR) was performed by ACROBiosystems. The Fc tagged ACE2 (AC2-H5257, ACROBiosystems) was immobilised in a Series S Sensor Chip CM5 (Cytiva) on a Biacore T200 (Cytiva) using the Human Antibody Capture Kit (Cytiva). The affinity measure was obtained using 8 different RBD heterodimer concentrations. The antigen structure simulations were performed with UCSF ChimeraX (Pettersen et al., 2021). 
bioRxiv preprint doi: https://doi.org/10.1101/2021.11.22.469117; this version posted December 4, 2021. The copyright holder for this

\subsection{SARS-CoV-2 recombinant protein RBD heterodimer adjuvanted vaccines}

The purified RBD fusion heterodimer was formulated with an oil in water adjuvant, equivalent to MF59C.1. The PHH-1V vaccine was tested at different concentrations: $0.04 \mu \mathrm{g}, 0.2 \mu \mathrm{g}, 1 \mu \mathrm{g}, 5$ $\mu \mathrm{g}$ and $20 \mu \mathrm{g}$ of RBD fusion heterodimer/dose for the safety and immunogenicity assays in $\mathrm{BALB} / \mathrm{c}$ mice. For efficacy assessment in the K18-hACE2 mice animal model, the vaccine was tested at $10 \mu \mathrm{g}$ and $20 \mu \mathrm{g}$ of fusion heterodimer/dose. The placebo vaccines were prepared with Phosphate Buffered Saline (PBS).

\subsection{Animal study design}

BALB/C mice (Envigo) and transgenic B6.Cg-Tg(K18-ACE2)2Prlmn/J (K-18-hACE2) (Jackson Laboratories) were used as animal models. All procedures that involved BALB/c mice were conducted in accordance with the European Union Guidelines for Animal Welfare (Directive 2010/63/EU) and approved by the Ethics Committee of HIPRA Scientific S.L.U. and the Department of Territori $i$ Sostenibilitat of the Catalan Government (file: 11388). The experimental procedure that involved the use of K18-hACE2 mice were conducted in accordance with the European Union Guidelines for Animal Welfare (Directive 2010/63/EU) and was approved by the CMCiB Ethics Committee and the Department of Territori i Sostenibilitat of the Catalan Government (file: 11490). The animal study design followed the principles of the 3R's and animal welfare.

Seventy-two $(48 \mathrm{~F}+24 \mathrm{M}) 5$-week-old BALB/c mice were allocated to 6 groups $(n=12 ; 8 \mathrm{~F}+4 \mathrm{M})$ and were used for safety and immunogenicity assays. Group A was vaccinated with PBS; group $B$ was immunised with $0.04 \mu \mathrm{g}$ recombinant protein RBD fusion heterodimer/dose; group C was immunised with $0.2 \mu \mathrm{g}$ recombinant protein RBD fusion heterodimer/dose; group D was immunised with $1 \mathrm{\mu g}$ recombinant protein RBD fusion heterodimer/dose; group E was immunised with $5 \mu \mathrm{g}$ recombinant protein RBD fusion heterodimer/dose; and group F was immunised with $20 \mu \mathrm{g}$ recombinant protein RBD fusion heterodimer/dose. These animals were monitored daily for clinical signs and bodyweight was recorded weekly until D35/D37; at that time, the animals were euthanised and tissues were collected. Animals were fed and watered ad libutum.

For further safety, immunogenicity, and efficacy assays, $62(31 \mathrm{~F}+31 \mathrm{M}) 4 / 5$-week-old K18humanised ACE2 (hACE2) mice were allocated to 4 groups ( $n=18 ; 9 F+9$ M except for the placebo group $n=8 ; 4 F+4 M)$. Specifically, group $A$ was intramuscularly injected with PBS and noninfected, group B was injected with PBS and infected with SARS-CoV-2; group C was vaccinated with $10 \mu \mathrm{g} /$ dose of recombinant protein RBD fusion heterodimer and infected with SARS-CoV2; and group D was vaccinated with $20 \mu \mathrm{g} /$ dose of recombinant protein RBD fusion heterodimer and infected with SARS-CoV-2. Animals from satellite subgroups were euthanised at D35 in order to assess the immunological response of the vaccinated group. Challenged animals were chronologically euthanised at D37, D39 and D42 (males)/D43 (females). Several tissue samples were collected for further analyses. Animal were fed and watered ad libutum.

\subsection{Mice vaccination and SARS-CoV-2 challenge}

In order to comply with animal welfare regulations, all animals were injected with $0.1 \mathrm{~mL} /$ dose and they were distributed equally in both hind legs $(2 \times 50 \mu \mathrm{L})$. Animals from satellite subgroups were euthanized at D35 in order to assess the immunological response of the vaccinated group. Vaccines were injected intramuscularly following a two-dose prime-and-boost schedule: $1^{\text {st }}$ dose (prime) at D0 and $2^{\text {nd }}$ dose (boost) at D21. The SARS-CoV-2 challenge was performed through intranasal inoculation on a subset of animals at D35 with $25 \mu \mathrm{L}$ in each nostril $\left(10^{3} \mathrm{TCID}_{50} / \mathrm{mice}\right.$ in $50 \mu \mathrm{L} /$ mice). The intranasal experimental infection was performed under sedation with isoflurane $4-5 \%$. BALB/c vaccination and sampling were performed at HIPRA (Girona, Spain). 
bioRxiv preprint doi: https://doi.org/10.1101/2021.11.22.469117; this version posted December 4, 2021. The copyright holder for this preprint (which was not certified by peer review) is the author/funder. All rights reserved. No reuse allowed without permission.

K18-hACE2 mice vaccination, SARS-CoV-2 challenge, and sampling were performed in the ABSL3 unit of the Comparative Medicine and Bioimage Centre of Catalonia of the Germans Trias i Pujol Research Institute (Badalona, Spain). The protocol followed is depicted in Figure S5.

\subsection{Analysis of SARS-CoV-2-specific antibodies}

Serum binding antibodies against SARS-CoV-2 RBD were determined by ELISA (HIPRA). MaxiSorp plates (Nunc, Roskilde, Denmark) were coated with $100 \mathrm{ng} /$ well RBD protein (Sino Biologicals, Beijing, China) and blocked with 5\% non-fat dry milk (Sigma-Aldrich, St. Louis, MO, USA) in PBS. Wells were incubated with serial dilutions of the serum samples and the bound total IgG specific antibodies were detected by Peroxidase-conjugated Goat Anti-Mouse IgG (Sigma-Aldrich). Finally, wells were incubated with K-Blue Advanced Substrate (Nirco, Madrid, Spain) and the absorbance at $450 \mathrm{~nm}$ was measured using a microplate reader (Synergy HTX). The mean value of the absorbance was calculated for each dilution of the serum sample run in duplicate. Isotypes IgG1 and IgG2a were detected using peroxidase affinipure goat anti-mouse IgG, Fcy subclass 1 specific and peroxidase affinipure Goat anti-mouse IgG, Fcy subclass 2a specific (Jackson ImmunoResearch, Cambridgeshire, UK), respectively, as a secondary antibody. The end-point titre of RBD-specific total IgG binding antibodies was established as the reciprocal of the last serum dilution that gave 3 times the mean optical density of the negative control of the technique (wells without serum added).

\subsection{Analysis of SARS-CoV-2 neutralising antibodies by pseudovirus neutralization assay}

Neutralising antibodies in serum against SARS-CoV-2 Wuhan (original sequence), and the alpha, beta, gamma and delta variants were determined by a pseudoviruses-based neutralization assay (PBNA) at IRSICaixa (Barcelona, Spain), using an HIV reporter pseudovirus that expresses the $S$ protein of SARS-CoV-2 and luciferase. Pseudovirus were generated as described in Pradenas et al., 2021. For the neutralization assay, 200 TCID50 of pseudovirus supernatant was preincubated with serial dilutions of the heat-inactivated serum samples for $1 \mathrm{~h}$ at $37^{\circ} \mathrm{C}$ and then added onto ACE2 overexpressing HEK293T cells. After $48 \mathrm{~h}$, cells were lysed with britelite plus luciferase reagent (PerkinElmer, Waltham, MA, USA). Luminescence was measured for $0.2 \mathrm{~s}$ with an ensight multimode plate reader (PerkinElmer). The neutralization capacity of the serum samples was calculated by comparing the experimental RLU calculated from infected cells treated with each serum to the max RLUs (maximal infectivity calculated from untreated infected cells) and min RLUs (minimal infectivity calculated from uninfected cells), and expressed as the neutralisation percentage: Neutralisation (\%) $=($ RLUmax-RLUexperimental)/(RLUmax-RLUmin) * 100 (Trinité et al., 2021). IC 50 were calculated by plotting and fitting neutralization values and the lo fog plasma dilution to a 4-parameters equation in Prism 9.0.2 (GraphPad Software, USA).

\subsection{Intra-cellular cytokine staining (ICS)}

The ICS was performed by the Infection Biology group, Department of Experimental and Health Sciences, Universitat Pompeu Fabra (DCEXS-UPF, Barcelona, Spain). Spleens from female mice were mechanically disrupted onto a $40 \mu \mathrm{M}$ cell strainer and incubated in $5 \mathrm{~mL}$ of $0.15 \mathrm{M}$ ammonium chloride buffer for $5 \mathrm{~min}$ at room temperature (RT) for red blood cell lysis. Cells were then washed in RPMI (Gibco, Tavarnuzze, Italy) supplemented with 10\% FBS, $1 \%$ penicillin/streptomycin, $0.05 \mathrm{mM}$-Mercaptoethanol and $1 \mathrm{mM}$ sodium pyruvate (cRPMI). Two million splenocytes per well (96-well plate) were stimulated in vitro under three conditions: (i) a 1:1 mix of the peptide libraries (PepMix ${ }^{\mathrm{TM}}$ ) from the B.1.1.7 ( $\alpha$ variant) and B.1.351 ( $\beta$ variant) lineages covering the entire RBD of the SARS-CoV-2 S protein; (ii) CRPMI (negative control); and (iii) PMA + lonomycin (positive control) for $5 \mathrm{~h}$ at 37C 5\% CO2 in cRPMI in the presence of Brefeldin A (Sigma-Aldrich) for the last $3 \mathrm{~h}$ before antibody staining. The final concentrations used were $1 \mu \mathrm{g} / \mathrm{mL}$ of each peptide of the RBD peptide pool, $15 \mathrm{ng} / \mathrm{mL}$ of PMA (Sigma-Aldrich) and $250 \mathrm{ng} / \mathrm{mL}$ of ionomycin (Sigma-Aldrich). For flow cytometric analysis, equal numbers of 
cells were stained with fixable viability stain 780 (BD Biosciences, New Jersey, NJ, USA) in PBS for 15 min at RT followed by staining with antibodies against CD3, CD4, CD8 and CD44 for 20 min on ice in FACS buffer (PBS 5\% FCS, 0.5\% BSA, 0.07\% NaN3). Cells were then fixed for 20 min on ice with $2 \%$ Formaldehyde and stained with antibodies against intracellular proteins (IFN $\gamma$, TNFa, IL-2 and IL-4) for 20 min on ice in perm/wash buffer (PBS 1\% FCS, NaN3 0.1\%, Saponin 0.1\%). All antibodies were purchased from either BD Biosciences, ThermoFisher or BioLegend (See Table S1 for more details). Samples were processed on an Aurora (Cytek, Fremont, CA, USA) analyser. FACS data were analysed using flowjo 10 software (Tree Star Inc., Ashland, OR, USA). The stain index was calculated by subtracting the mean fluorescence intensity (MFI) of the unstained or fluorescence minus one (FMO) control from the MFI of the stained samples and dividing it by two times the standard deviation of the unstained population. Background cytokine expression in the no-peptide (CRPMI) condition was subtracted from that measured in the RBD peptide pool for each mouse.

\subsection{Mouse cytokine assay}

The cytokine assay was performed by the Infection Biology group, Department of Experimental and Health Sciences, Universitat Pompeu Fabra (DCEXS-UPF, Barcelona, Spain). Splenocytes from female mice were seeded at $1.1 \times 10^{6}$ cells/well in 24-well plates and stimulated with a 1:1 mix of the RBD overlapping peptides from B.1.1.7 (alpha variant) and B.1.1351 (beta variant) lineages $(1 \mu \mathrm{g} / \mathrm{mL}$ each). cRPMI media was used as a negative control and PMA (15 ng/mL) + lonomycin $(250 \mathrm{ng} / \mathrm{mL})$ as a positive control. The supernatants were harvested after $48 \mathrm{~h}$ incubation at $37^{\circ} \mathrm{C}$ and a panel that quantifies the cytokines IL-2, IL-4, IL-5, IL-6, IL-10, IFN- $\gamma$ and TNF- $\alpha$ (Luminiex Multiplex, Invitrogen, Waltham, MA, USA) was run according to the manufacturer's instructions. These measurements were performed at Servei Bioquímica Veterinària, Facultat de Veterinària, Universitat Autònoma de Barcelona (UAB, Barcelona, Spain).

\subsection{IFN- $\gamma$ and IL-4 ELISpot assays}

ELISpot assays were performed with mouse IFN- $\gamma$ and IL-4 ELISpot PLUS kits according to the manufacturer's instructions (3321-4HPT-10 and 3311-4HPW-10, Mabtech, Herndon, VA, USA). A total of $2,5 \times 10^{5}$ or $4 \times 10^{5}$ splenocytes from female mice were seeded per well for the IFN- $\gamma$ and IL-4 tests respectively and ex vivo stimulated either with the 1:1 mix of the RBD overlapping peptides from the B.1.1.7 (alpha variant) and B.1.351 (beta variant) lineages (1 $\mu \mathrm{g} / \mathrm{mL}$ each), or with complete cRPMI (negative control) or with Concanavalin A $(5 \mu \mathrm{g} / \mathrm{ml}$ (Sigma-Aldrich)) (positive control). Each condition was run in duplicates. After an incubation period of 18-20 $\mathrm{h}$ (for IFN- $\gamma$ ) or $48 \mathrm{~h}$ (for IL-4), the plates were manipulated according to the manufacturer's instructions. Spots were counted under a dissection microscope. Frequencies of IFN- $\gamma$ or IL-4secreting cells were expressed as the number of responding cells per million splenocytes. The number of spots in unstimulated cultures (negative control) was subtracted from the spot count in RBD-stimulated cultures.

\subsection{SARS-CoV-2 genomic RT-qPCR}

Total viral load in respiratory tissue samples was determined by RT-qPCR (CReSA, IRTA-UAB, Barcelona, Spain), according to the method described by Brustolin et al. (2021).

\subsection{Virus titration}

Virus titres were determined using a standard TCID ${ }_{50}$ assay in Vero E6 cells at CReSA (IRTA-UAB), according to the method described by Brustolin et al., 2021. $\mathrm{TCID}_{50} / \mathrm{ml}$ results were transformed to PFU/ml units by multiplying $T C I D_{50} / \mathrm{ml}$ titre by 0.7 according to the ATCC (ATCC Technical Support recommendations, 2021). To express viral titres in PFU/g, lungs and nasal turbinate 
bioRxiv preprint doi: https://doi.org/10.1101/2021.11.22.469117; this version posted December 4, 2021. The copyright holder for this

samples were determined at necropsy on days $2,4,7$ or 8 post-infection (and at the time the euthanasia was required).

\subsection{Histopathology}

Histopathological analyses were performed at CReSA (IRTA-UAB). Upper (nasal turbinate) and lower (lung) respiratory tract and brain were fixed in $10 \%$ buffered formalin and routinary processed for histopathology. Haematoxylin- and eosin-stained slides were examined under optical microscope. Multifocal broncho-interstitial pneumonia, multifocal lymphoplasmacytic rhinitis and non-suppurative meningoencephalitis were evaluated from: lung nasal turbinate and brain lesions, respectively, according to the following score: 0 (no lesion), 1 (mild lesion), 2 (moderate lesion) and 3 (severe lesion) (Brustolin et al., 2021; Vidal et al., 2021).

\subsection{Statistical analysis}

All statistical analyses were performed using R (version 4.0.5), SPSS (version 22), and/or GraphPad Prism (version 9). In all plots, individual data points were depicted for each mouse along with the sample mean and Standard Deviation (SD) as a representation of their distribution. Significant comparisons at $5 \%$ significance level and trends were indicated in each plot ( $\left.p<0.05:{ }^{*} ; p<0.01{ }^{* *} ; 0.05<p<0.1:+\right)$. For testing the effect of a single factor, ANOVA was used when normality could be considered. Data violating the assumption of normality was analysed using Kruskal-Wallis' test. Only when the assumption of homoscedasticity was not satisfied, was Welch's ANOVA performed. For pairwise comparisons, the corresponding posthoc tests were chosen depending on the nature of the data and the planned comparisons to perform. For comparisons against zero-variance groups (all observations having the same value), one-sample t-tests were employed (labelled in each plot with a "1"). Finally, only in some cases, a priori pairwise comparisons were performed using two-sample specific tests such as unpaired $t$, Mann-Whitney's $U$, and Fisher's Exact tests. Survival analyses (Kaplan-Meier estimates and log-rank test to compare groups) were performed to study differences in clinical signs and mortality. Linear mixed effects models were used to analyse repeated measures variables. Time, treatment and their interaction were considered as potential fixed factors and the experimental subject was considered a random factor. Random intercept models were fitted to the data and restricted maximum likelihood was used for estimation. Model assumptions were tested graphically, and correlation between longitudinal data as well as heteroscedasticity was modelled using the nlme package in R. For pairwise comparisons, estimated marginal means were calculated and compared using the emmeans package in R, correcting for multiple testing using the multivariate t-distribution adjustment.

Supplementary Materials: Supplementary data are provided in the enclosed document. This includes: Figure S1. SARS-CoV-2 RBD protein-specific IgG responses in K18-hACE2 mice. Figure S2. Neutralising antibody responses by PBNA (original sequence from Wuhan) in K18-hACE2 mice. Figure S3. Frequencies of PHH-1V-induced Th1 responses in $\mathrm{CD}^{+}$and $\mathrm{CD} 8^{+} \mathrm{T}$ cells. Figure S4. Bodyweight monitoring during the vaccination period in mice. Figure S5. Schematic representation of the experimental protocol in BALB/c and K18-hACE2 mice for the safety, immunogenicity, and efficacy assessment. Table S1. Th1/Th2 CD4/CD8 cytokine markers used for ICS.

Author Contributions: ${ }^{1}$ HIPRA: Promoted and designed the studies; designed the vaccine antigen and formulation, and produced the vaccine batches; performed the animal procedures in the study with BALB/c mice; performed the ELISA to determine the IgG titres against RBD. ${ }^{2,3,4}$ CReSA: Challenge SARS-CoV-2 strain preparation, performed the RT-qPCR, virus titrations and histological analysis in the efficacy study in K18-hACE2 mice. ${ }^{5,6} \mathrm{IrsiCaixa}$ : Analyses of the neutralising antibodies against SARS-CoV-2 by PBNA. ${ }^{7} \mathrm{CMCiB}-I G T P$ : Performed the SARS- 
CoV-2 challenge and animal procedures in the efficacy study in K18 hACE2 mice. ${ }^{8,9}$ UPF:/ICREA Analyses of vaccine-induced cellular immune responses.

Acknowledgements: We would like to thank Marta Ribó, Ariadna Pararols, Rubén Hernández and Helena Sánchez for their assistance in the animal care; Jèssica Gómez and Maria Bosch for their support in the tabulation of raw data; Anna Moya, Mireia Muntada, Lidia Galindo and Núria Elias for the ELISA analysis and protein characterization; Manuel Cañete for his support in the statistical analysis; Marta Bau for their assistance in the production of the vaccine antigen; Claudia Millán for helping with the modelling and visualization of the heterodimeric RBD structure; David Solanes Foz for his support to the ABSL3 Unit; the CMCiB Ethical Committee members (Jorge Carrillo, Eulàlia Genescà, Carol Galvez, Carol Soler, and Patricia Fachal) for their support on the ethical review of animal procedures; the IGTP Biosafety Committee members (Pere-Joan Cardona, Julià Blanco, Meritxell Carrió, Natalia Ruiz, David Izquierdo, and Noemí Parraga) for their support on the review biosafety procedures; Glòria Pujol and Eduard Fossas for their assistance in the revision of the manuscript; and Francisco Díaz-Sáez and Anna Muñoz, from Alta Medical Services, for providing medical writing support. We gratefully acknowledge editorial support of Sarah Marshall.

Funding: This project was partially funded by the Centre for the Development of Industrial Technology (CDTI), a public organisation answering to the Spanish Ministry of Science and Innovation.

Competing Interests: Authors indicated as "1" are employees of HIPRA, a private pharmaceutical company that develops and manufactures vaccines. CReSA, IrsiCaixa, CMCiBIGTP, UPF and ICREA have received financial support from HIPRA.

Disclosure Statements: Several patent applications have been filed by HIPRA SCIENTIFIC S.L.U. and Laboratorios HIPRA, S.A. on different SARS-CoV-2 vaccine candidates and SARS-CoV-2 subunit vaccines, including the novel recombinant RBD fusion heterodimer PHH-1V. These patent applications are not yet public. Antonio Barreiro, Antoni Prenafeta, Luis González, Laura Ferrer, Esther Puigvert, Jordi Palmada, Maria Teresa Prat and Carmen Garriga are the inventors of these patent applications

\section{References}

Alanagreh, L., Alzoughool, F. and Atoum, M. (2020) "The Human Coronavirus Disease COVID-19: Its Origin, Characteristics, and Insights into Potential Drugs and Its Mechanisms," Pathogens, 9(5), p. 331. doi:10.3390/pathogens9050331.

An, Y. et al. (2021) "A tandem-repeat dimeric RBD protein-based COVID-19 vaccine ZF2001 protects mice and nonhuman primates," bioRxiv, p. 2021.03.11.434928. doi:10.1101/2021.03.11.434928.

ATCC Technical Support remommendations 2021). Available at: https://www.atcc.org/support/technical-support/faqs/converting-tcid-50-to-plaque-formingunits-pfu. (access date: 16 November 2021).

Brustolin, M. et al. (2021) "Protection against reinfection with D614- or G614-SARS-CoV-2 isolates in golden Syrian hamster," Emerging Microbes \& Infections, 10(1), pp. 797-809. doi:10.1080/22221751.2021.1913974. 
Comirnaty SmPC 2021 (2021) "https://www.ema.europa.eu/en/documents/productinformation/comirnaty-epar-product-information_en.pdf."

COVID-19 Vaccine Janssen (2021) available https://www.ema.europa.eu/en/documents/product-information/covid-19-vaccine-janssenepar-product-information_en.pdf.

Dai, L. and Gao, G.F. (2021) "Viral targets for vaccines against COVID-19," Nature Reviews Immunology, 21(2), pp. 73-82. doi:10.1038/s41577-020-00480-0.

Francica, J.R. et al. (2021) "Vaccination with SARS-CoV-2 Spike Protein and AS03 Adjuvant Induces Rapid Anamnestic Antibodies in the Lung and Protects Against Virus Challenge in Nonhuman Primates.," BioRxiv : [Preprint]. doi:10.1101/2021.03.02.433390.

Garcia-Beltran, W.F. et al. (2021) "Multiple SARS-CoV-2 variants escape neutralization by vaccine-induced humoral immunity," Cell, 184(9), pp. 2372-2383.e9. doi:10.1016/j.cell.2021.03.013.

Gavi Alliance- Covax collaboration platform (2021) "What are protein subunit vaccines and how could they be used against COVID-19?"

Genomic epidemiology of novel coronavirus - Global subsampling. (2021) Available at: https://nextstrain.org/ncov/gisaid/global?d=frequencies\&p=full. (access date: 16 November 2021).

Goepfert, P.A. et al. (2021) "Safety and immunogenicity of SARS-CoV-2 recombinant protein vaccine formulations in healthy adults: interim results of a randomised, placebo-controlled, phase 1-2, dose-ranging study," The Lancet Infectious Diseases, 21(9), pp. 1257-1270. doi:10.1016/S1473-3099(21)00147-X.

Günl, F. et al. (2021) "Shooting at a moving target-effectiveness and emerging challenges for sars-cov-2 vaccine development," Vaccines. MDPI. doi:10.3390/vaccines9101052.

Hadfield, J. et al. (2018) "Nextstrain: real-time tracking of pathogen evolution," Bioinformatics, 34(23), pp. 4121-4123. doi:10.1093/bioinformatics/bty407.

Hannah Ritchie et al. (2020) "Coronavirus Pandemic (COVID-19)," published online at OurWorldInData.org. (access date: 16 November 2021)

Huang, Y. et al. (2020) "Structural and functional properties of SARS-CoV-2 spike protein: potential antivirus drug development for COVID-19," Acta Pharmacologica Sinica, 41(9), pp. 1141-1149. doi:10.1038/s41401-020-0485-4.

Jangra, S. et al. (2021) "SARS-CoV-2 spike E484K mutation reduces antibody neutralisation," The Lancet Microbe, 2(7), pp. e283-e284. doi:10.1016/S2666-5247(21)00068-9.

Keech, C. et al. (2020a) "Phase 1-2 Trial of a SARS-CoV-2 Recombinant Spike Protein Nanoparticle Vaccine," New England Journal of Medicine, 383(24), pp. 2320-2332. doi:10.1056/NEJMoa2026920.

Keech, C. et al. (2020b) "Phase 1-2 Trial of a SARS-CoV-2 Recombinant Spike Protein Nanoparticle Vaccine," New England Journal of Medicine, 383(24), pp. 2320-2332. doi:10.1056/nejmoa2026920. 
Kleanthous, H. et al. (2021) "Scientific rationale for developing potent RBD-based vaccines targeting COVID-19," npj Vaccines, 6(1), p. 128. doi:10.1038/s41541-021-00393-6.

Ko, E.-J. and Kang, S.-M. (2018) "Immunology and efficacy of MF59-adjuvanted vaccines," Human Vaccines \& Immunotherapeutics, 14(12), pp. 3041-3045. doi:10.1080/21645515.2018.1495301.

Kuo, T.-Y. et al. (2020) "Development of CpG-adjuvanted stable prefusion SARS-CoV-2 spike antigen as a subunit vaccine against COVID-19," Scientific Reports, 10(1), p. 20085. doi:10.1038/s41598-020-77077-z.

Kyriakidis, N.C. et al. (2021) "SARS-CoV-2 vaccines strategies: a comprehensive review of phase 3 candidates," npj Vaccines, 6(1), p. 28. doi:10.1038/s41541-021-00292-w.

Lee, W.S. et al. (2020) "Antibody-dependent enhancement and SARS-CoV-2 vaccines and therapies," Nature Microbiology, 5(10), pp. 1185-1191. doi:10.1038/s41564-020-00789-5.

Li, T. et al. (2020) "SARS-CoV-2 spike produced in insect cells elicits high neutralization titres in non-human primates," Emerging Microbes \& Infections, 9(1), pp. 2076-2090. doi:10.1080/22221751.2020.1821583.

Mathieu, E. et al. (2021) "Author Correction: A global database of COVID-19 vaccinations," Nature Human Behaviour, 5(7), pp. 956-959. doi:10.1038/s41562-021-01160-2.

Moyo-Gwete, T. et al. (2021) "SARS-CoV-2 501Y.V2 (B.1.351) elicits cross-reactive neutralising antibodies," bioRxiv, p. 2021.03.06.434193. doi:10.1101/2021.03.06.434193.

O'Hagan, D.T. et al. (2013) "The history of MF59 " adjuvant: a phoenix that arose from the ashes," Expert Review of Vaccines, 12(1), pp. 13-30. doi:10.1586/erv.12.140.

Pallesen, J. et al. (2017) "Immunogenicity and structures of a rationally designed prefusion MERS-CoV spike antigen," Proceedings of the National Academy of Sciences, 114(35), pp. E7348E7357. doi:10.1073/pnas.1707304114.

Pettersen, E.F. et al. (2021) "UCSF ChimeraX": Structure visualization for researchers, educators, and developers," Protein Science, 30(1), pp. 70-82. doi:10.1002/pro.3943.

Piccoli, L. et al. (2020) "Mapping Neutralising and Immunodominant Sites on the SARS-CoV-2 Spike Receptor-Binding Domain by Structure-Guided High-Resolution Serology," Cell, 183(4), pp. 1024-1042.e21. doi:10.1016/j.cell.2020.09.037.

Planas, D. et al. (2021) "Reduced sensitivity of SARS-CoV-2 variant Delta to antibody neutralization," Nature, 596(7871), pp. 276-280. doi:10.1038/s41586-021-03777-9.

Plante, J.A. et al. (2021) "The variant gambit: COVID-19's next move," Cell Host and Microbe. Cell Press, pp. 508-515. doi:10.1016/j.chom.2021.02.020.

Pradenas, E. et al. (2021) "Stable neutralising antibody levels 6 months after mild and severe COVID-19 episodes," Med, 2(3), pp. 313-320.e4. doi:10.1016/j.medj.2021.01.005.

Ramanathan, M. et al. (2021) "SARS-CoV-2 B.1.1.7 and B.1.351 spike variants bind human ACE2 with increased affinity," The Lancet Infectious Diseases, 21(8), p. 1070. doi:10.1016/S14733099(21)00262-0. 
Sharker, S.Md. and Rahman, A. (2021) "A Review on the Current Methods of Chinese Hamster Ovary (CHO) Cells Cultivation for the Production of Therapeutic Protein," Current Drug Discovery Technologies, 18(3), pp. 354-364. doi:10.2174/1570163817666200312102137.

Singh, M., and O'Hagan, D. (1999) Advances in vaccine adjuvants. Nat Biotechn, 17, pp. 10751081. doi.org/10.1038/15058

Snapper, C.M. and Paul, W.E. (1987) "Interferon- $y$ and B Cell Stimulatory Factor-1 Reciprocally Regulate Ig Isotype Production," Science, 236(4804), pp. 944-947. doi:10.1126/science.3107127.

Spellberg, B. and Edwards, J.E. (2001) "Type 1/Type 2 Immunity in Infectious Diseases," Clinical Infectious Diseases, 32(1), pp. 76-102. doi:10.1086/317537.

Spikevax SmPC (2021) available at: https://www.ema.europa.eu/en/documents/productinformation/spikevax-previously-covid-19-vaccine-moderna-epar-product-information_en.pdf.

Tian, J.-H. et al. (2021) "SARS-CoV-2 spike glycoprotein vaccine candidate NVX-CoV2373 immunogenicity in baboons and protection in mice," Nature Communications, 12(1), p. 372. doi:10.1038/s41467-020-20653-8.

Trinité, B. et al. (2021) "SARS-CoV-2 infection elicits a rapid neutralising antibody response that correlates with disease severity," Scientific Reports, 11(1), p. 2608. doi:10.1038/s41598-02181862-9.

U.S. National Library of Medicine (2021) "Safety and Immunogenicity Study of Recombinant Protein RBD Candidate Vaccine Against SARS-CoV-2 in Adult Healthy Volunteers (COVID-19). Identifier: NCT05007509. available at: https://www.clinicaltrials.gov/ct2/show/NCT05007509."

Vidal, C. et al. (2021) "Chronological brain lesions after SARS-CoV-2 infection in hACE2transgenic mice," Veterinary Pathology, accepted (2021).

Vogel, A.B. et al. (2020) "A prefusion SARS-CoV-2 spike RNA vaccine is highly immunogenic and prevents lung infection in non-human primates," bioRxiv, p. 2020.09.08.280818. doi:10.1101/2020.09.08.280818.

Wall, E.C. et al. (2021) "Neutralising antibody activity against SARS-CoV-2 VOCs B.1.617.2 and B.1.351 by BNT162b2 vaccination," The Lancet, 397(10292), pp. 2331-2333. doi:10.1016/S01406736(21)01290-3.

Wang, H. et al. (2020) "SARS-CoV-2 Proteome Microarray for Mapping COVID-19 Antibody Interactions at Amino Acid Resolution," ACS Central Science, 6(12), pp. 2238-2249. doi:10.1021/acscentsci.0c00742.

WHO (2021) WHO Coronavirus (COVID-19) Dashboard, available at: https://covid19.who.int/ (access date: 16 November 2021).

$\mathrm{Yi}, \mathrm{C}$. et al. (2020) "Key residues of the receptor binding motif in the spike protein of SARS-CoV2 that interact with ACE2 and neutralising antibodies," Cellular \& Molecular Immunology, 17(6), pp. 621-630. doi:10.1038/s41423-020-0458-z.

Zhou, P. et al. (2020) "A pneumonia outbreak associated with a new coronavirus of probable bat origin," Nature, 579(7798), pp. 270-273. doi:10.1038/s41586-020-2012-7. 


\section{Figures}
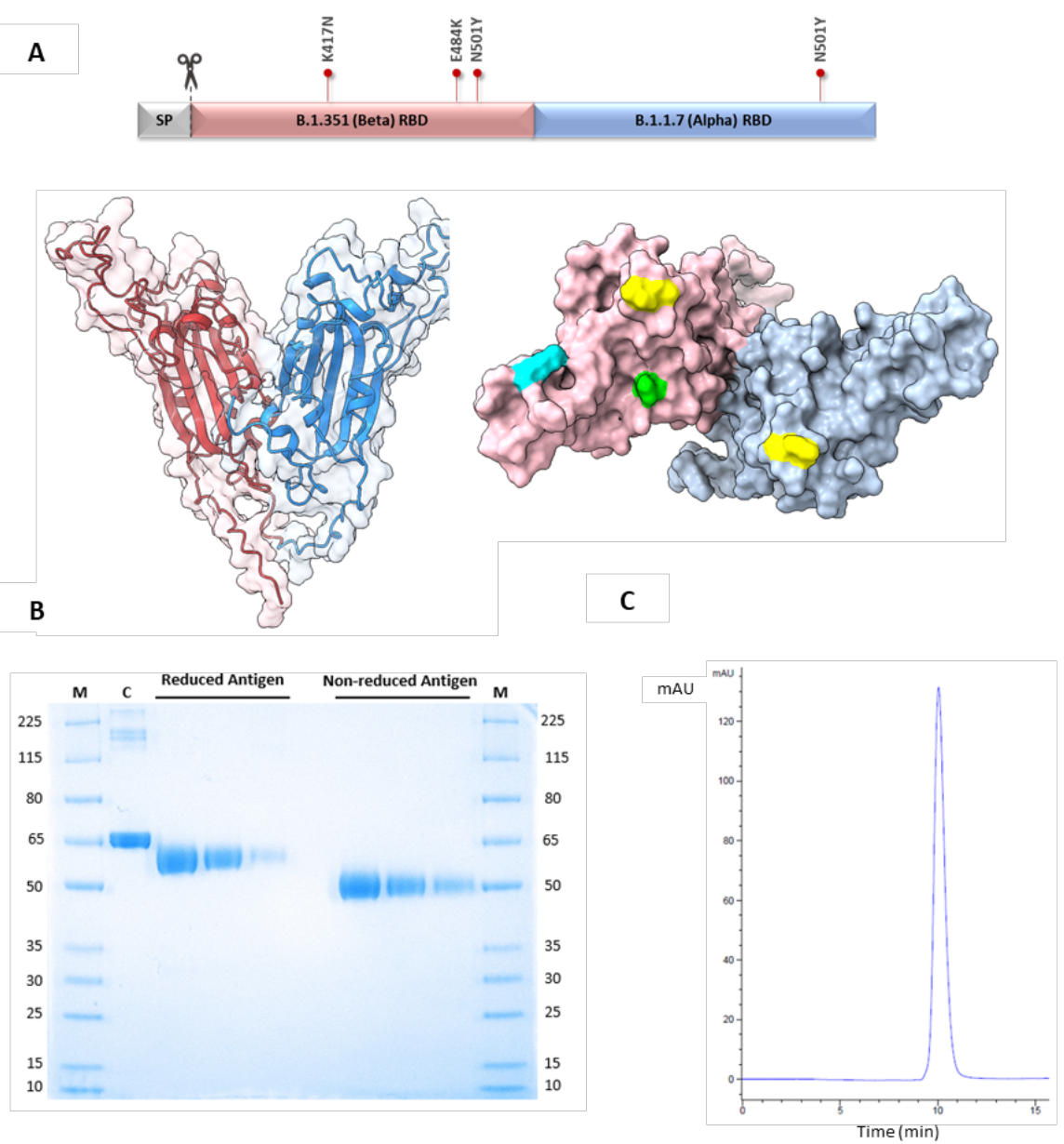

D

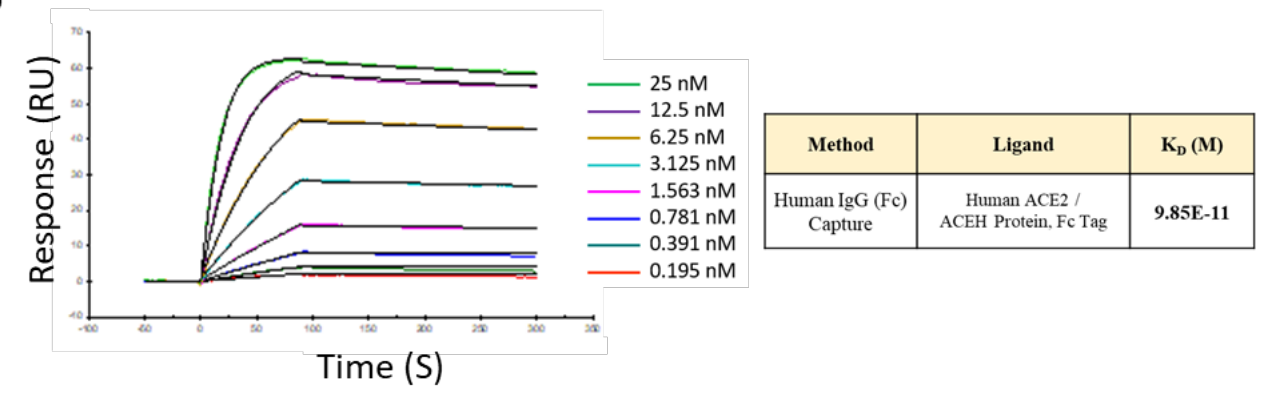

Figure 1. Structure and characterisation of the B.1.351 (Beta) - B.1.1.7 (Alpha) RBD receptor-binding domain (RBD) heterodimer, immunogen of $\mathrm{PHH}-1 \mathrm{~V}$. (A) Structural representation of the RBD heterodimer. Top: sequence diagram. Bottom left: front view of the RBD heterodimer cartoon structure. Bottom right: top view of the antigen surface structure. Mutations are highlighted in green (K417N), cyan (E484K) and yellow (N501Y). (B) SDS-PAGE. The reduced and non-reduced purified antigens were loaded at three serial dilutions: $1 / 10,1 / 20$ and 1/40. M: molecular weight ladder. C: BSA control. (C) SEC-HPLC chromatogram of the purified antigen. (D) Surface plasmon resonance (SPR) for the quantitative evaluation of the affinity between the antigen and its natural ligand, the human ACE2 receptor. RU: resonance units. 

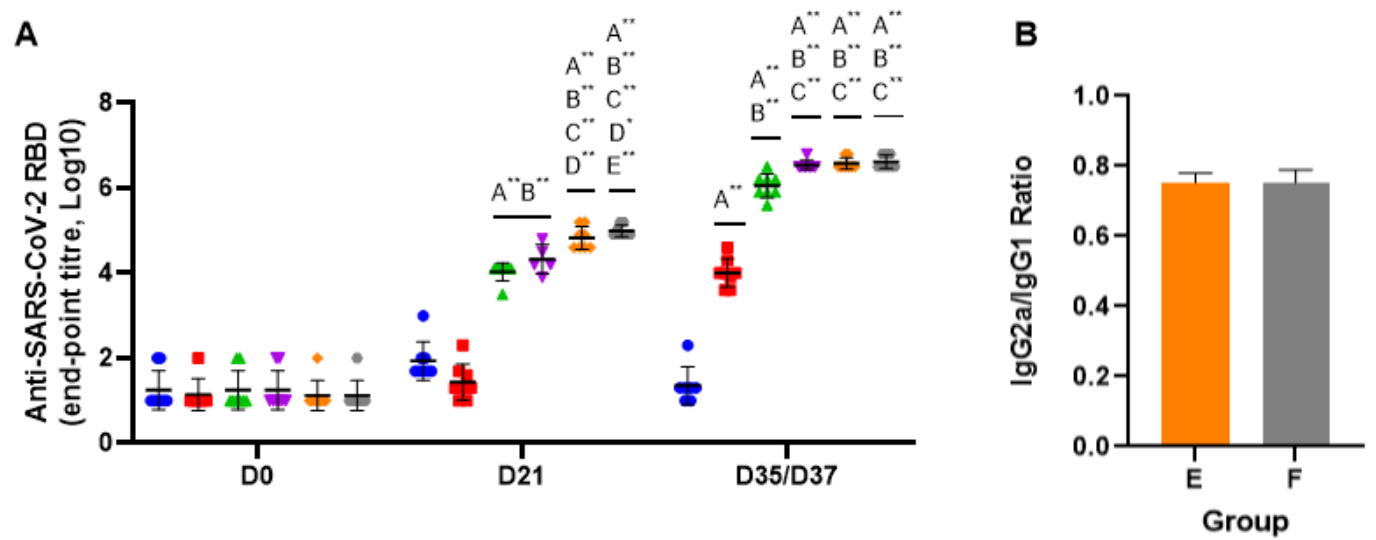

- A: Control (PBS)

- B: PHH-1V $(0.04 \mu \mathrm{g}$ RBD fusion dimer/dose $)$

E: PHH-1V (5 $\mu \mathrm{g}$ RBD fusion dimer/dose)

$\Delta \quad$ C: $\mathrm{PHH}-1 \mathrm{~V}(0.2 \mu \mathrm{g}$ RBD fusion dimer/dose $)$

F: PHH-1V (20 $\mu \mathrm{g}$ RBD fusion dimer/dose)

v D: PHH-1V (1 ug RBD fusion dimer/dose)

- E: PHH-1V $(5 \mu \mathrm{g}$ RBD fusion dimer/dose)

- F: PHH-1V (20 $\mu \mathrm{g}$ RBD fusion dimer/dose)

Figure 2. Analysis of the immunogenicity of the PHH-1V vaccine in mice. (A) SARS-CoV-2 RBD-specific IgG responses in groups $A$ to $F$ on days D0, D21 and D35/D37. End-point antibody titres determined by ELISA are shown. Data from female BALB/c mice are shown. Group A $(n=8)$, vaccinated with PBS as a control; group $B(n=8)$, immunized with $0.04 \mu$ recombinant protein RBD fusion heterodimer/dose; group $C(n=8)$, immunized with $0.2 \mu \mathrm{g}$ recombinant protein RBD fusion heterodimer/dose; group $D(n=8)$, immunized with $1 \mu \mathrm{g}$ recombinant protein RBD fusion heterodimer/dose; group $E(n=8)$, immunized with $5 \mu \mathrm{g}$ recombinant protein RBD fusion heterodimer/dose; and group $F(n=8)$, immunized with $20 \mu \mathrm{g}$ recombinant protein RBD fusion heterodimer/dose. Each data point represents an individual mouse serum, with bars representing the mean titre and the standard deviation (SD). Statistically significant differences between groups are indicated with a line on top of each group: ${ }^{*} p<0.05 ; * *<0.01$. (B) Endpoint titre ratios of IgG2a to IgG1 in female mice vaccinated with $\mathrm{PHH}-1 \mathrm{~V}$ vaccine (groups $\mathrm{E}$ and $\mathrm{F}$ ). Analyses of IgG1 and IgG2 subclasses in groups $E$ and $F$ were performed by ELISA on serum samples taken on day D35/D37. Data represent mean ratio \pm SD. 


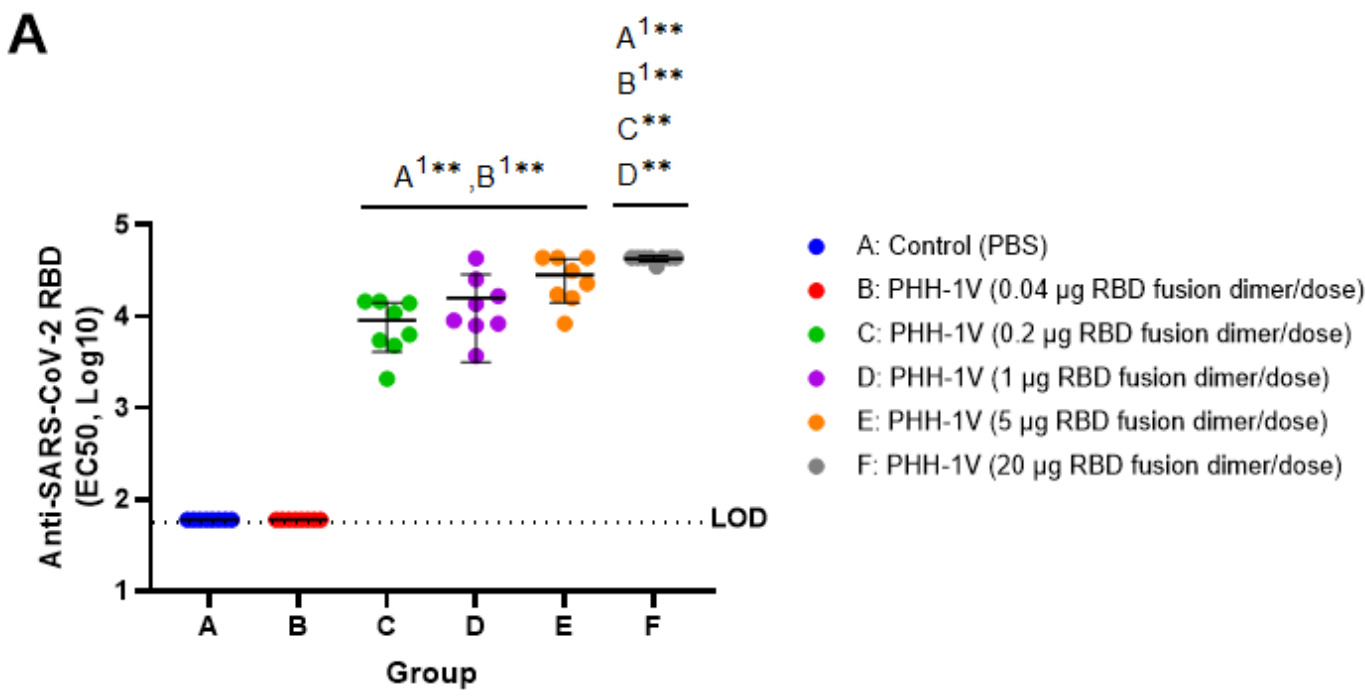

B

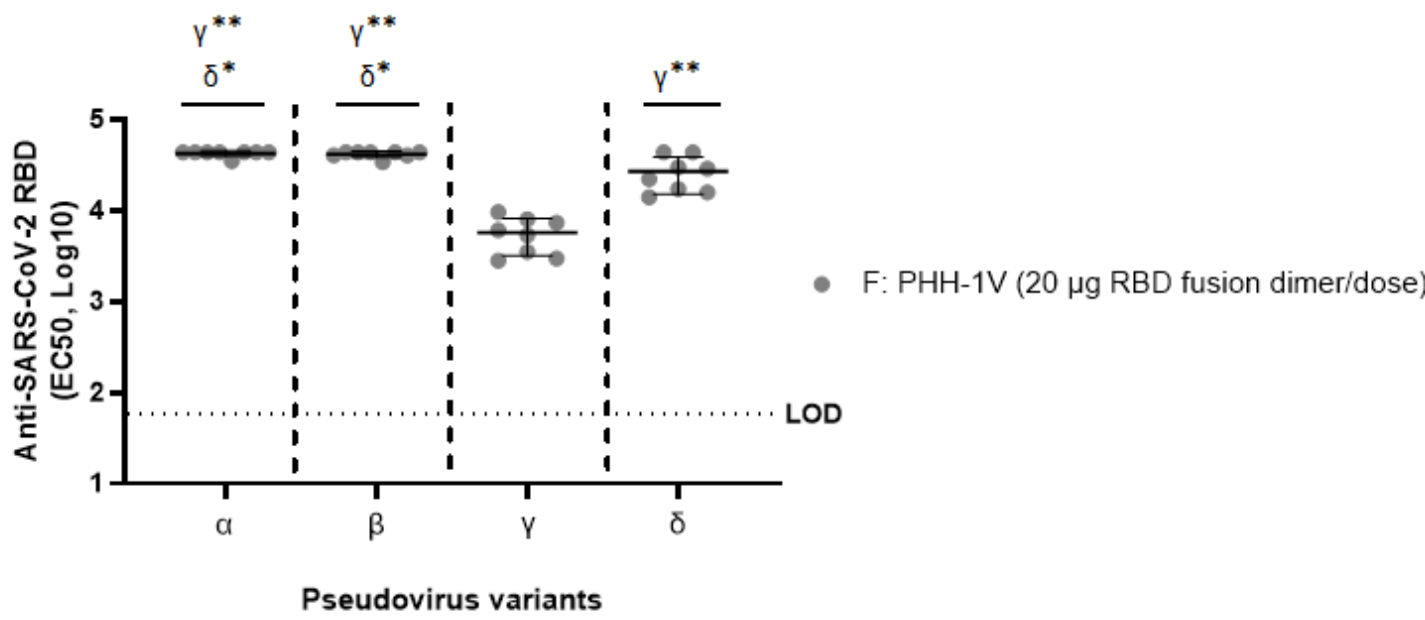

Figure 3. Neutralising antibody response in groups $A$ to $F$ upon vaccination. (A) Neutralising antibody responses in groups A to F. SARS-CoV-2 neutralising antibody titres in sera, against pseudoviruses that express the $S$ protein with the $\alpha$ sequence, were determined by PBNA at 14/16 days after the second dose of each vaccine (D35/D37). Sera from female BALB/c mice collected on D35/D37 were assessed for pseudovirus-neutralising activity. Titres are expressed as $\log _{10} I C 50$. Group $A(n=8)$, vaccinated with PBS as a control; group B $(n=8)$, immunized with $0.04 \mu \mathrm{g}$ recombinant protein RBD fusion heterodimer/dose; group $C(n=8)$, immunized with $0.2 \mu$ g recombinant protein RBD fusion heterodimer/dose; group $D(n=8)$, immunized with $1 \mu \mathrm{g}$ recombinant protein RBD fusion heterodimer/dose; group $\mathrm{E}(\mathrm{n}=8)$, immunized with $5 \mu \mathrm{g}$ recombinant protein RBD fusion heterodimer/dose; and group $F(n=8)$, immunized with $20 \mu \mathrm{g}$ recombinant protein RBD fusion heterodimer/dose. (B) Neutralising antibody responses against multiple SARS-CoV- 2 variants by PBNA $(\alpha, \beta, \gamma, \delta)$ upon $20 \mu \mathrm{g}$ RBD fusion heterodimer/dose immunization. Sera from group $F$ mice collected at D35/D37 were assessed for pseudovirus-neutralising activity. Titres are expressed as $\log _{10} E C 50$. Each data point represents an individual mouse serum, with bars representing the median titre \pm SD. Statistically significant differences between groups are indicated with a line on top of each group: ${ }^{*} p<0.05 ;{ }^{* *} p<0.01$. Comparisons against zero-variance groups (all observations having the same value) are indicated as: 1 . One-sample t-tests were employed for these comparisons. Data from female mice are shown. 
A
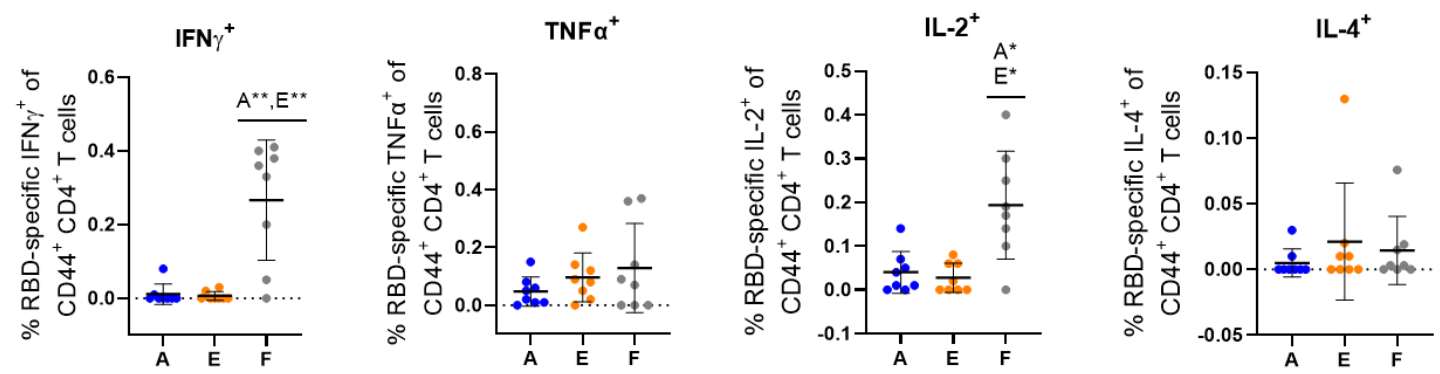

B

IFN $\gamma^{+}$

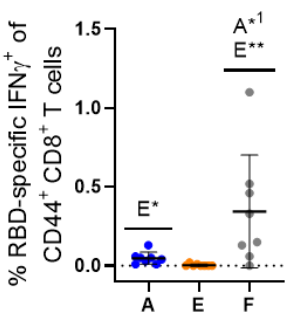

$\mathrm{TNFa}^{+}$

$\mathrm{IL}-\mathbf{2}^{+}$

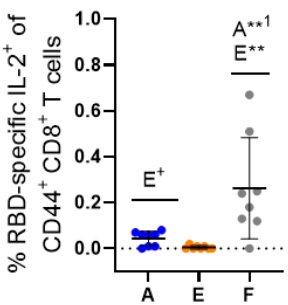

- A: Control (PBS)

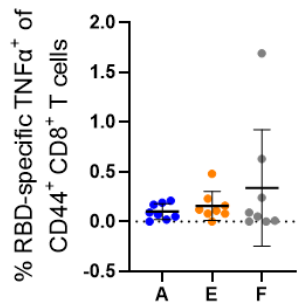

E: PHH-1V (5 $\mu \mathrm{g}$ RBD fusion dimer/dose)

F: PHH-1V $(20 \mu \mathrm{g}$ RBD fusion dimer/dose)

Figure 4. PHH-1V-induced CD4 and CD8 T cell responses. Splenocytes from vaccinated BALB/c mice were isolated 14-16 days after boost immunization (D35/D37), stimulated with RBD peptide pools, and analysed by intracellular cytokine staining. The frequencies of cytokine expressing CD4 T cells (A) and CD8 T cells (B) are shown. The cytokine expression in splenocytes stimulated with the medium was considered the background value and this was subtracted from peptide-specific responses. Each data point represents an individual mouse. Bars represent the mean per group \pm SD. Group $A(n=8$; controls), immunized with PBS; group $\mathrm{E}(\mathrm{n}=8)$, immunized with $5 \mu \mathrm{g} /$ dose of PHH-1V; and group F $(n=8)$, immunized with $20 \mu \mathrm{g} / \mathrm{dose}$ of $\mathrm{PHH}-1 \mathrm{~V}$. Statistically significant differences between groups are indicated with a line on top of the groups: ${ }^{*} p<0.05 ;{ }^{* *} p<0.01$. ${ }^{1}$ One-sample t-tests were employed for these comparisons. Data from female mice are shown. 
INFY

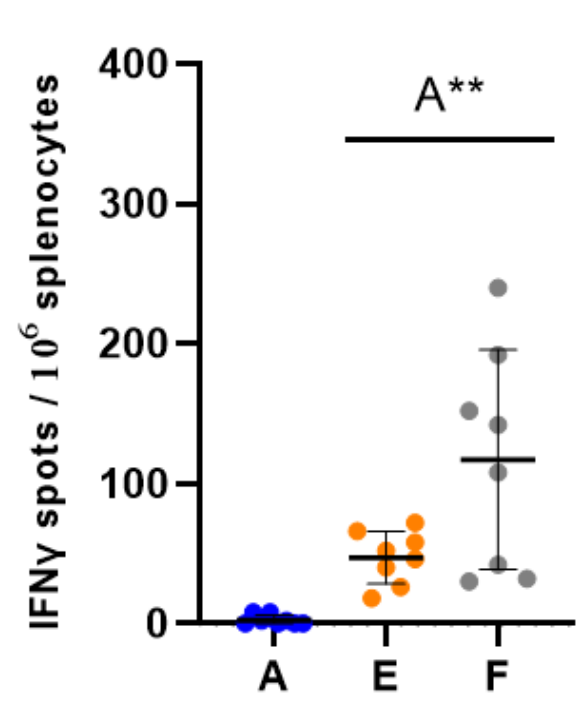

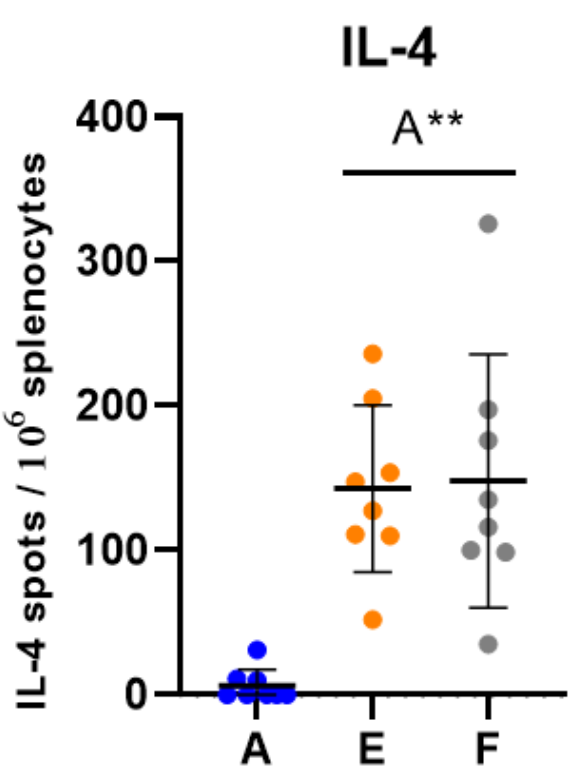

A $E$

- A: Control (PBS)

- $\mathrm{E}: \mathrm{PHH}-1 \mathrm{~V}(5 \mu \mathrm{g}$ RBD fusion dimer/dose)

- $\mathrm{F}: \mathrm{PHH}-1 \mathrm{~V}(20 \mu \mathrm{g}$ RBD fusion dimer/dose)

Figure 5. Frequencies of PHH-1V-induced IFN-g and IL-4 responses. Splenocytes from vaccinated BALB/C mice were isolated 14-16 days after boost immunization (D35/D37), stimulated with RBD peptide pools and analysed by IFN-g- and IL-4-specific ELISpot assays. Group A ( $n=8$; controls), immunized with PBS; group $\mathrm{E}(\mathrm{n}=8)$, immunized with $5 \mu \mathrm{g} /$ dose of $\mathrm{PHH}-1 \mathrm{~V}$; and group $\mathrm{F}(\mathrm{n}=8)$, immunized with $20 \mu \mathrm{g} / \mathrm{dose}$ of $\mathrm{PHH}-1 \mathrm{~V}$. Each data point represents an individual mouse. Bars represent the mean per group \pm SD. Statistically significant differences between groups are indicated with a line on top of each group. ** $p<0.01$. Data from female mice are shown. 
IFN-Y

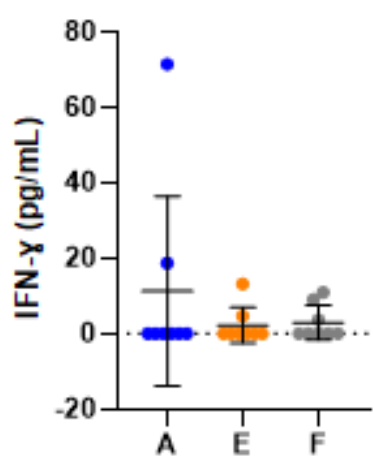

IL-2

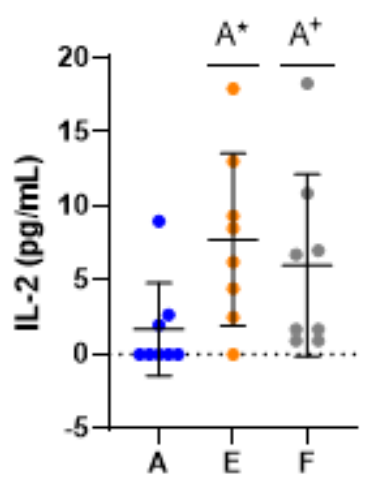

IL-4

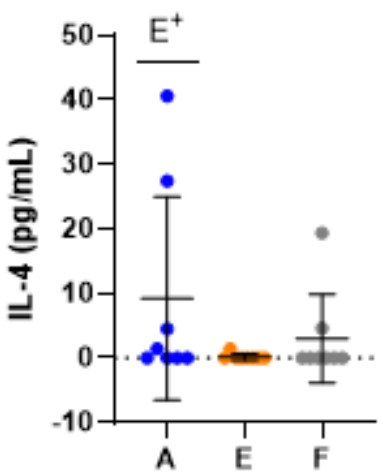

\section{IL-5}

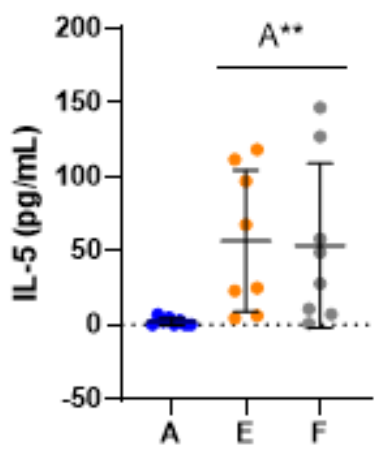

IL-10

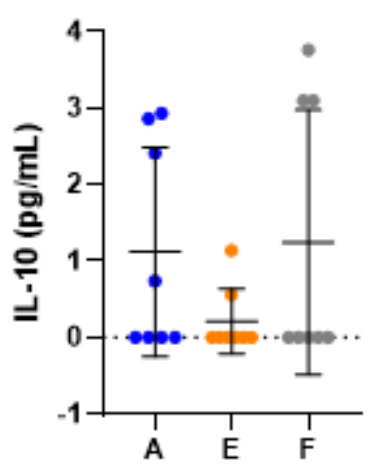

- A: Control (PBS)
E: PHH-1V (5 $\mu \mathrm{g}$ RBD fusion dimer/dose)
IL-6

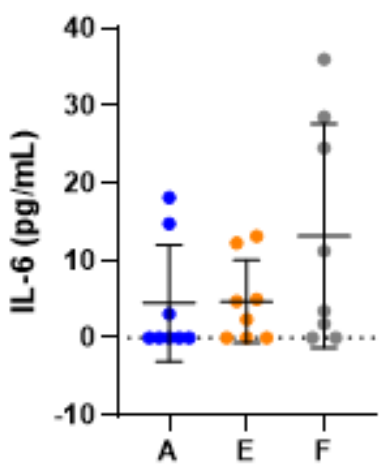

TNF- $\alpha$

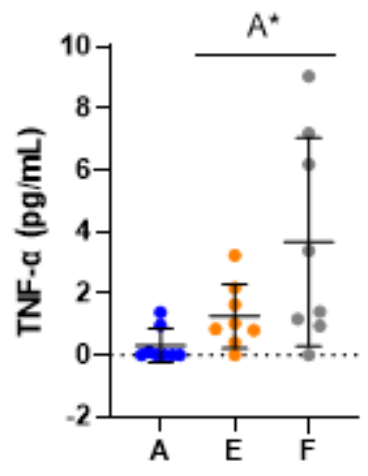

F: PHH-1V $(20 \mu g$ RBD fusion dimer/dose)

Figure 6. Extracellular cytokine levels in vaccine-induced splenocytes in mice at 14-16 days after boost immunization (D35/D37). Extracellular cytokines were measured by Luminex Multiplex in supernatants from BALB/C splenocytes stimulated with a pool of peptides from SARS-CoV-2 RBD. Cytokine levels in splenocytes stimulated with the medium were considered the background value and these were subtracted from the responses measured from the RBD peptide pool for each individual mouse. Group A $(n=8)$, vaccinated with PBS as a control group; group $E(n=8)$, immunized with $5 \mu \mathrm{g} /$ dose of recombinant protein RBD fusion heterodimer in oil-based adjuvant; and group $F(n=8)$, immunized with $20 \mu \mathrm{g} / \mathrm{dose}$ of recombinant protein RBD fusion heterodimer in oil-based adjuvant. Each data point represents an individual mouse with bars representing the mean \pm SD. Statistically significant differences between groups in the expression of a cytokine are indicated with a line on top of each group: ${ }^{*} p<0.05 ; * * p<0.01$. $+: 0.05<p<0.1$. Data from female mice are shown. 
bioRxiv preprint doi: https://doi.org/10.1101/2021.11.22.469117; this version posted December 4, 2021. The copyright holder for this preprint (which was not certified by peer review) is the author/funder. All rights reserved. No reuse allowed without permission.

A

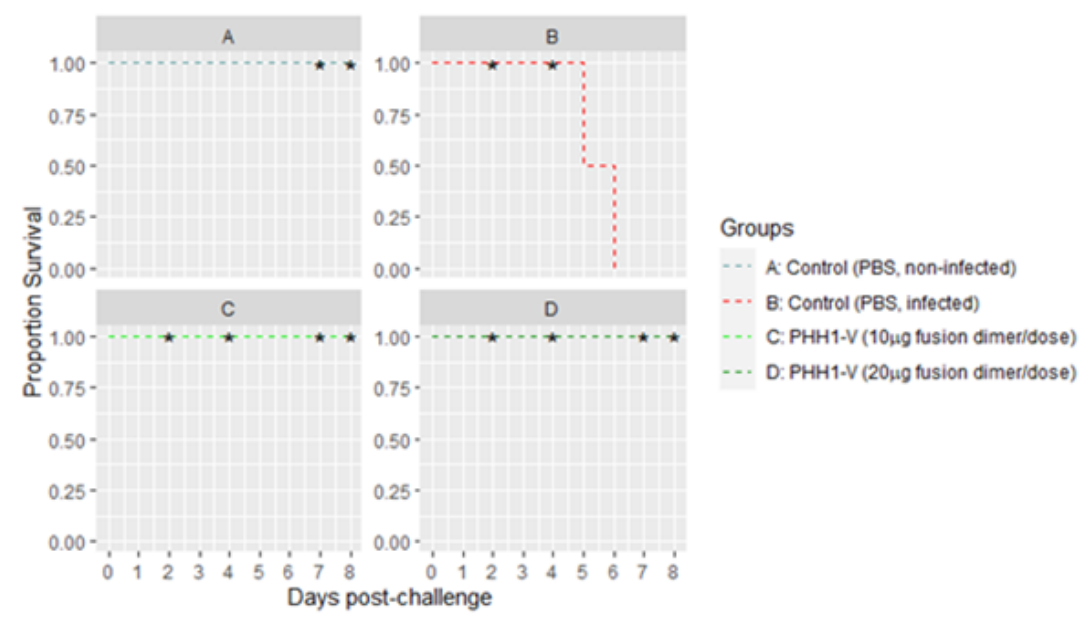

B

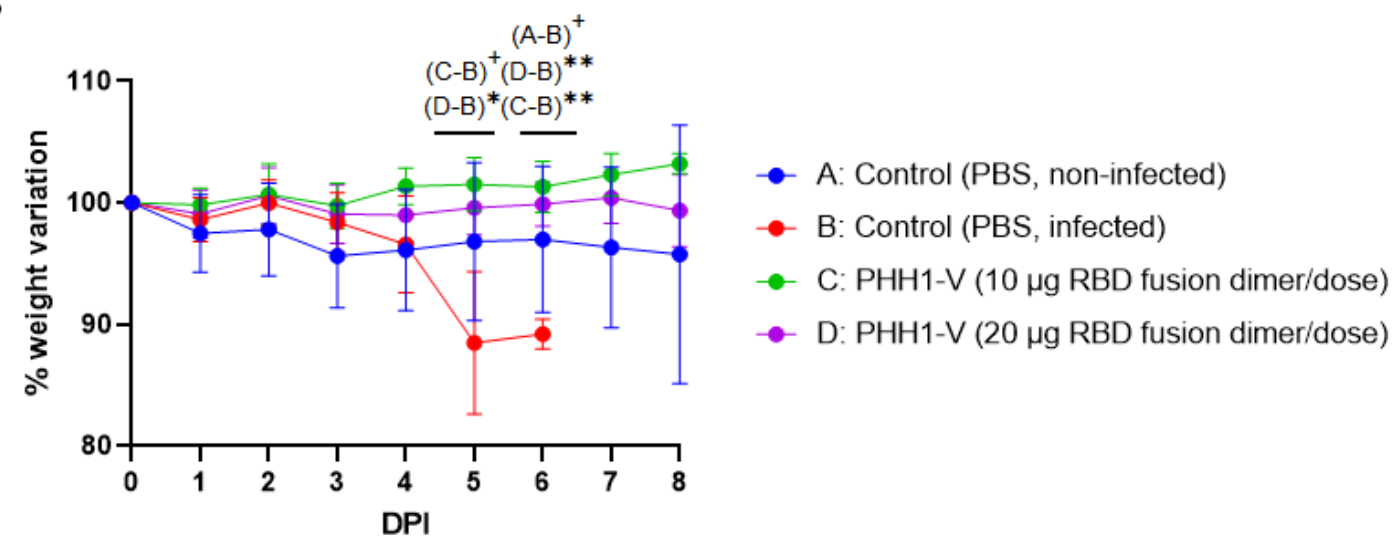

Figure 7. Survival and bodyweight loss in vaccinated and non-vaccinated mice upon SARS-CoV-2 challenge. (A) Survival curves of groups immunized K18-hACE2 mice with PHH-1V vaccine and control groups. Group A, vaccinated with PBS and non-infected $(n=8,4 F+4 M)$; group $B$, vaccinated with PBS and infected with SARS-CoV-2 $(n=18,9 F+9 M)$; group C, vaccinated with $10 \mu \mathrm{g} /$ dose of recombinant protein RBD fusion heterodimer in oil-based adjuvant and infected with SARS-CoV-2 $(n=18,9 F+9 M)$; and group $D$, vaccinated with $20 \mu \mathrm{g} /$ dose of recombinant protein RBD fusion heterodimer in oil-based adjuvant and infected with SARS-CoV-2 $(n=18,9 F+9 M)$. Asterisks represent censored data (i.e., animals leaving the study; in this case, it represents selected animals that were euthanized for serial necropsies). (B) Mean weight change after SARS-CoV-2 challenge calculated as a percentage of the pre-challenge weight in K18hACE2 mice. Group A, vaccinated with PBS and non-infected $(n=8,4 F+4 M)$; group B: vaccinated with PBS and infected with SARS-CoV-2 $(\mathrm{n}=18,9 \mathrm{~F}+9 \mathrm{M})$; group $\mathrm{C}$, vaccinated with $10 \mu \mathrm{g} / \mathrm{dose}$ of recombinant protein RBD fusion heterodimer in oil-based adjuvant and to be infected with SARS-CoV-2 ( $n=18,9 F+$ $9 \mathrm{M})$; and group $\mathrm{D}$, vaccinated with $20 \mu \mathrm{g} /$ dose of recombinant protein RBD fusion dimer in oil-based adjuvant and infected with SARS-CoV-2 $(n=18,9 F+9 M)$. Each data point represents the mean, with bars representing the SD. Statistically significant differences between groups in the expression of a cytokine are indicated with a line on top of each group: ${ }^{*} p<0.05 ;{ }^{* *} p<0.01$. $+: 0.05<p<0.1$. DPI: days postinfection. 
A

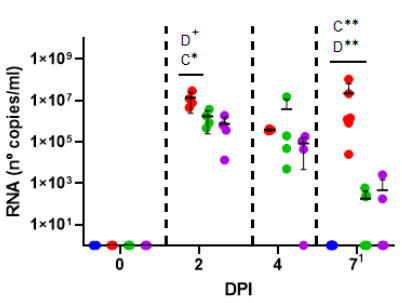

B
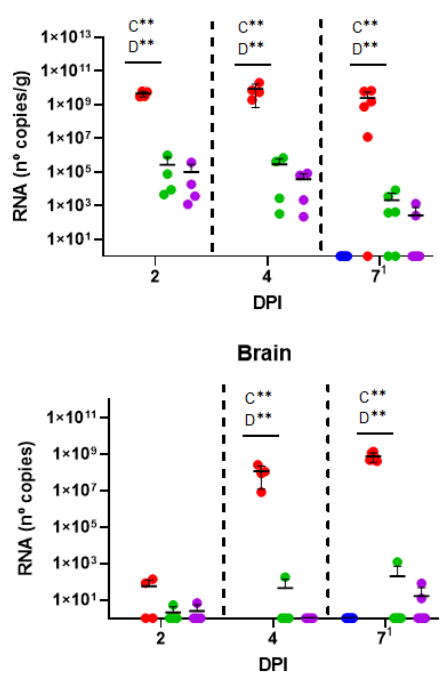

C

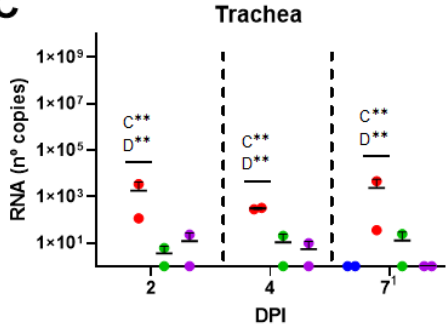

Spleen

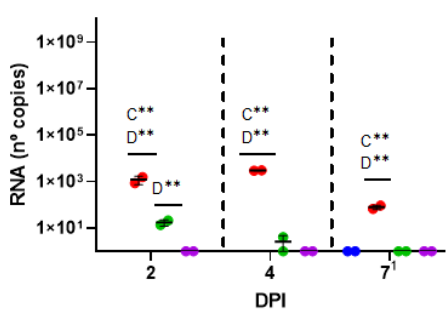

- A: Control (PBS, non-infected)

- B: Control (PBS, infected)
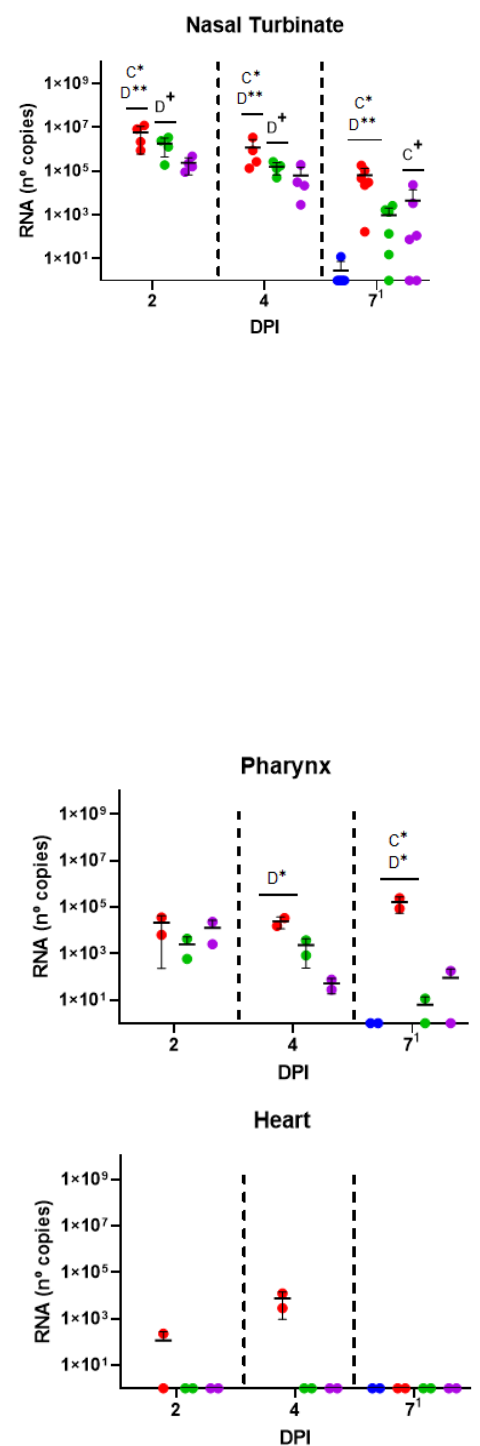

- C: $\mathrm{PHH}-1 \mathrm{~V}(10 \mu \mathrm{g}$ RBD fusion dimer/dose $)$

- D: PHH-1V (20 $\mu \mathrm{g}$ RBD fusion dimer/dose)

Figure 8. Viral load in different tissues from mice. (A) SARS-CoV-2 RT-qPCR detection in oropharyngeal swabs (number of copies/mL), collected from challenged mice. (B) SARS-CoV-2 RT-qPCR in lungs (number of copies/mL), nasal turbinate (number of copies), and brain (number of copies), collected from challenged animals. (C) SARS-CoV-2 RT-qPCR in trachea, pharynx, spleen, and heart in number of copies, collected in challenged animals. Each data point represents an individual mouse value, with bars representing the mean \pm SD. Statistically significant differences between groups in the expression of a cytokine are indicated with a line on top of each group: ${ }^{*} p<0.05 ;{ }^{*} p<0.01$. $+: 0.05<p<0.1$. DPI: days post-infection. 

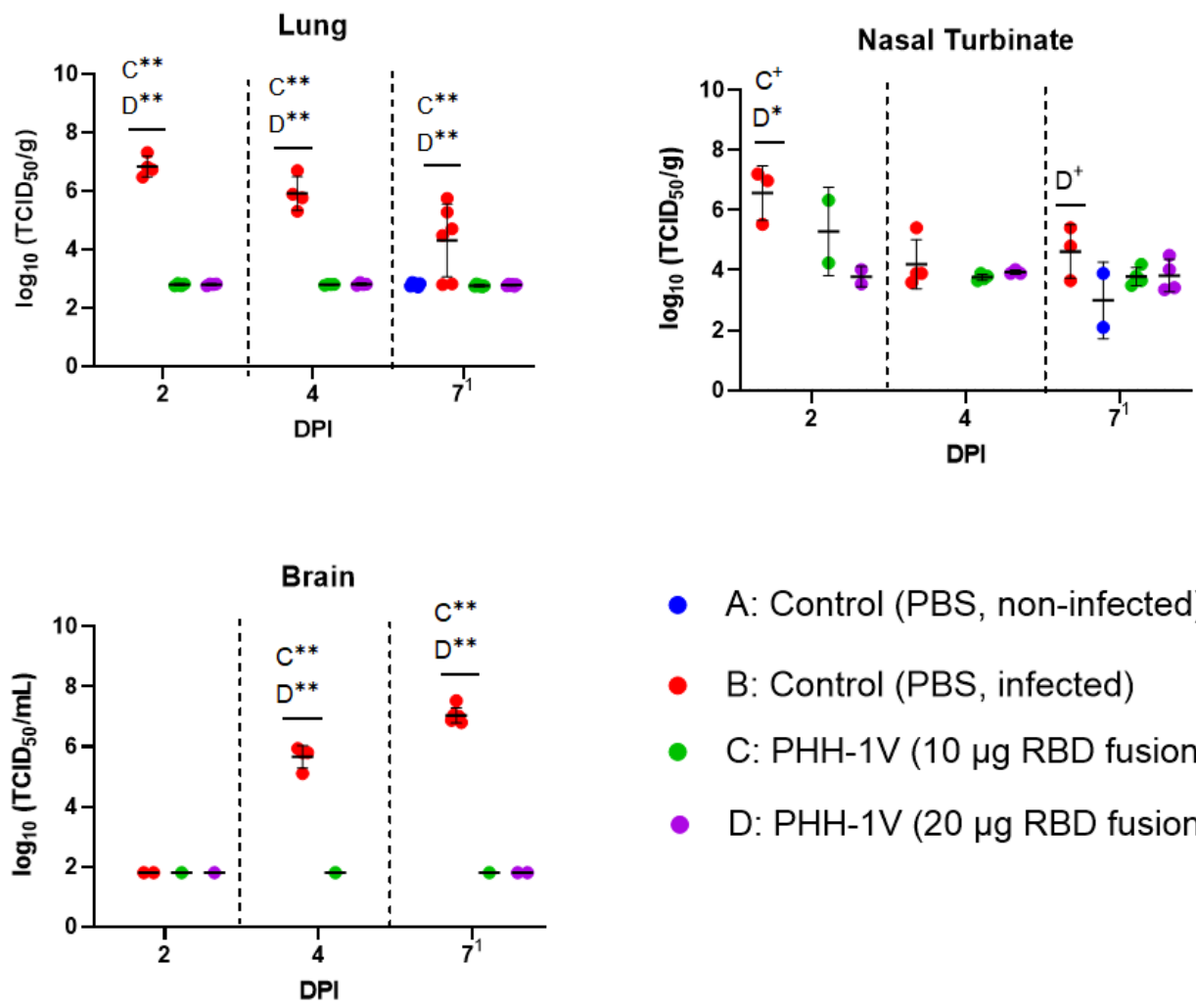

- A: Control (PBS, non-infected)

- B: Control (PBS, infected)

- C: PHH-1V (10 $\mu \mathrm{g}$ RBD fusion dimer/dose)

- D: PHH-1V (20 $\mu \mathrm{g}$ RBD fusion dimer/dose)

Figure 9. SARS-CoV-2 viral titration in Vero E6 cells from lung, nasal turbinate and brain samples $\left(\mathrm{TCID}_{50} / \mathrm{mL}\right)$ from mice. Virus titres were determined using a standard $\mathrm{TCID}_{50}$ assay on positive samples of RT-qPCR (in some exceptional cases, RT-qPCR and viral isolation were performed in parallel, for logistical reasons). Group A, vaccinated with PBS and non-infected; group B, vaccinated with PBS and infected with SARS-CoV-2; group $C$, vaccinated with $10 \mu \mathrm{g}$ /dose of recombinant protein RBD fusion heterodimer in oil-based adjuvant and infected with SARS-CoV-2; and group D, vaccinated with 20 $\mu \mathrm{g} /$ dose of recombinant protein RBD fusion heterodimer in oil-based adjuvant and infected with SARSCoV-2. The detection limit was set at $1.8 \mathrm{TCID}_{50} / \mathrm{mL}$. Samples scheduled on day 7 for groups $A, C$, and D correspond to D7 (for males) or D8 (for females); samples of group B were taken on D5 ( $=3$ ) or D6 ( $=3$ ), when animals reached the end-point criteria. Each data point represents an individual mice value, with bars representing the mean \pm SD. Statistically significant differences between groups are indicated with a line on top of each group: ${ }^{*} p<0.05 ;{ }^{*} p<0.01$. $+: 0.05<p<0.1$. DPI: days post-infection. 

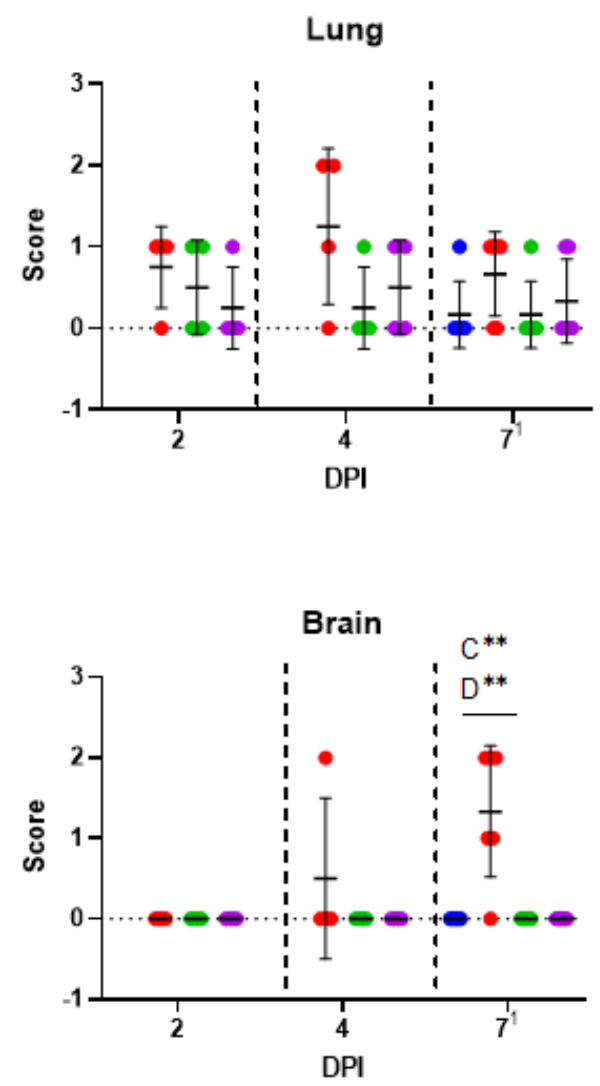

- A: Control, (PBS non-infected)

- B: Control, (PBS infected)

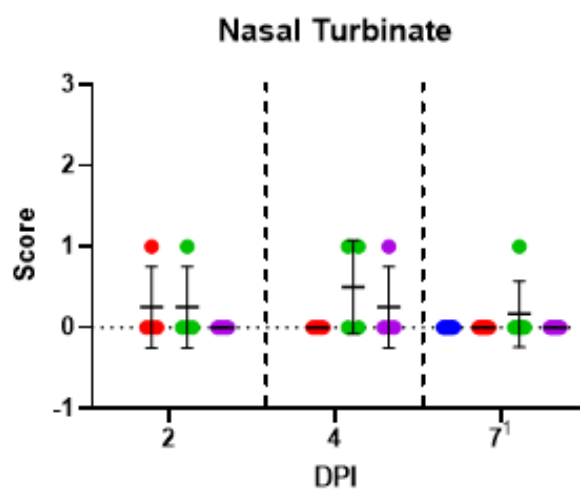

Hind limb skeletal muscle

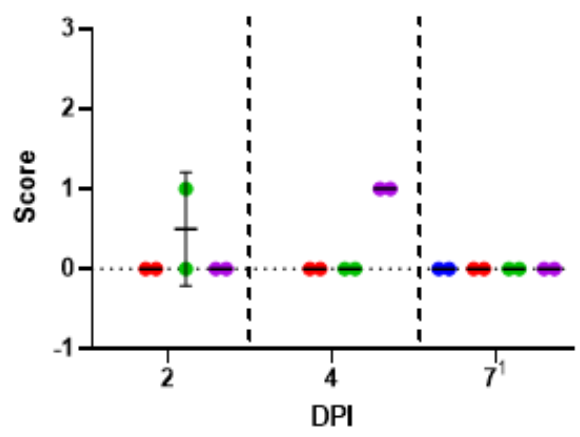

- C: PHH-1V (10 $\mu \mathrm{g}$ RBD fusion dimer/dose)

- D: PHH-1V (20 $\mu \mathrm{g}$ RBD fusion dimer/dose)

Figure 10. Histopathology score in lungs, nasal turbinate, brain, and hind limb skeletal muscle stained with H\&E from K18-hACE2 mice samples at end-point days. Histopathological analyses from the lung, nasal turbinate, and brain were determined for all animals, whereas hind limb skeletal muscle analysis, was determined for 2 animals per group. For each tissue sample, lesions were classified as follows: (A) multifocal broncho-interstitial pneumonia; (B) multifocal lymphoplasmacytic rhinitis; (C) multifocal lymphoplasmacytic meningoencephalitis; and (D) multifocal mononuclear inflammatory infiltrates within and around muscular fibres. Lesions were evaluated with the following score: 0 (no lesion); 1 (mild lesion); 2 (moderate lesion); and 3 (severe lesion). Samples scheduled for day 7 from groups A, C and D correspond to D7 (for males) or D8 (for females); samples of group B were taken on D5 $(n=3)$ or D6 $(n=3)$, when animals reached the end-point criteria. Each data point represents an individual mouse value, with bars representing the mean \pm SD. Statistically significant differences between groups are indicated with a line on top of each group: ${ }^{*} p<0.05 ; * *<<0.01$. DPI: days post-infection. 